\title{
The ferroptosis and iron-metabolism signature robustly predicts clinical diagnosis, prognosis and immune microenvironment for hepatocellular carcinoma
}

Jiansong Ji ( $\nabla$ Ischrjjs@163.com )

Lishui Central Hospital and Fifth Affiliated Hospital of Wenzhou Medical College https://orcid.org/0000-0002-6026-3676

\section{Bufu Tang}

Zhejiang University School of Medicine Second Affiliated Hospital

Jinyu Zhu

Zhejiang University School of Medicine Second Affiliated Hospital

Jie Li

Zhejiang University School of Medicine Second Affiliated Hospital

\section{Kai Fan}

Zhejiang University School of Medicine Second Affiliated Hospital

\section{Yang Gao}

Lishui Central Hospital and Fifth Affiliated Hospital of Wenzhou Medical College

\section{Shimiao Cheng}

Lishui Central Hospital and Fifth Affiliated Hospital of Wenzhou Medical College

\section{Chunli Kong}

Lishui Central Hospital and Fifth Affiliated Hospital of Wenzhou Medical College

\section{Liyun Zheng}

Lishui Central Hospital and Fifth Affiliated Hospital of Wenzhou Medical College

\section{Fazong Wu}

Lishui Central Hospital and Fifth Affiliated Hospital of Wenzhou Medical College

\section{Qiaoyou Weng}

Lishui Central Hospital and Fifth Affiliated Hospital of Wenzhou Medical College Chenying Lu

Lishui Central Hospital and Fifth Affiliated Hospital of Wenzhou Medical College

\section{Research}

Keywords: ferroptosis, hepatocellular carcinoma (HCC), TMB, immune microenvironment, prognosis 
Posted Date: March 31st, 2020

DOl: https://doi.org/10.21203/rs.3.rs-17980/v1

License: (c) (i) This work is licensed under a Creative Commons Attribution 4.0 International License. Read Full License 


\section{Abstract}

Background : In this study, we comprehensively analyzed genes related to ferroptosis and iron metabolism to construct diagnostic and prognostic models and explore the relationship with the immune microenvironment in HCC.

Methods : Integrated analysis, cox regression and the least absolute shrinkage and selection operator (LASSO) method of 104 ferroptosis- and iron metabolism-related genes and HCC-related RNA sequencing were performed to identify HCC-related ferroptosis and iron metabolism genes.

Results : four genes (ABCB6, FLVCR1, SLC48A1 and SLC7A11) were identified to construct prognostic and diagnostic models. Poorer overall survival (OS) was exhibited in the high-risk group than that in the lowrisk group in both the training cohort $(P<0.001, H R=0.27)$ and test cohort $(P<0.001, H R=0.27)$. The diagnostic models successfully distinguished HCC from normal samples and proliferative nodule samples. Compared with low-risk groups, high-risk groups had higher TMB; higher fractions of macrophages, follicular helper T cells, memory B cells, and neutrophils; and exhibited higher expression of CD83, B7H3, OX40 and CD134L. As an inducer of ferroptosis, erastin inhibited HCC cell proliferation and progression, and it was showed to affect Th17 cell differentiation and IL-17 signaling pathway through bioinformatics analysis, indicating it a potential agent of cancer immunotherapy.

Conclusions : The prognostic and diagnostic models based on the four genes indicated superior diagnostic and predictive performance, indicating new possibilities for individualized treatment of HCC patients.

\section{Background}

As the most frequent primary malignant tumor of the liver[1], hepatocellular carcinoma (HCC) is ranked as the sixth most commonly diagnosed neoplasm and is estimated to be the fourth leading cause of cancer-related death worldwide[2]. The incidence and mortality of HCC are continuing to increase[3]. Based on multiple staging systems, HCC has made great progress in diagnosis and treatment, but most HCC staging systems are currently based on tumor burden and stratification of the disease by prognosis[4]. These systems lack sensitivity and have difficulty explaining the adverse biological characteristics that affect treatment and survival response, which generally limits the treatment effect for patients[5]. HCC is a highly complex, multistep process involving genetic mutations, chromosomal aberrations, molecular signaling pathways, and epigenetic disorders[6]. Therefore, a better understanding of the molecular changes, molecular mechanisms and characterization involved in tumorigenesis and the identification of novel biomarkers that can individually predict the diagnosis and prognosis of tumors are essential for personalized medicine[7].

Iron is a basic nutrient element in the human body, and it is indispensable for biological processes such as cell metabolism, growth and proliferation[8]. The homeostasis of iron metabolism is stably regulated through balanced absorption, systemic transport, and cellular uptake and storage[9]. Alterations in iron 
metabolism have a dual effect on tumor cells. Since most tumor cells have an increased demand for iron, an increase in iron reserves within a certain range can promote the growth and proliferation of tumor cells[10; 11], and there is a positive correlation between the risk of tumors and iron accumulation[12; 13], but the excessive increase in iron concentration in the body leads to cell death caused by membrane lipid peroxidation, termed ferroptosis[14]. Ferroptosis has anticancer functions that are useful in cancer treatment[15]. Since the first demonstration in 2012, ferroptosis has received widespread attention as a potential therapeutic pathway for cancer treatment. Various studies have determined the key role of ferroptosis in killing tumor cells and inhibiting tumor growth[16; 17]. Some previous studies have also confirmed the important significance of ferroptosis for the treatment and prognosis of liver cancer[18; 19], but the detailed signal transduction pathways and key regulators of ferroptosis during the occurrence and progression of HCC are unclear.

As an emerging feature of cancer, TMB was first emphasized in next-generation sequencing analysis[20]. TMB is defined as the total number of somatic coding mutations associated with the emergence of new antigens that trigger antitumor immunity[21]. It is speculated that highly mutated tumors are more likely to carry neoantigens, making them targets for activated immune cells[22]. Currently, immune checkpoint inhibitor-based immunotherapy as an innovative therapy for multiple types of advanced cancer is emerging, and TMB has been identified as an emerging biomarker that is sensitive to immune checkpoint inhibitors[23]. TMB can help identify patients with some types of cancer that could benefit from immunotherapy. In addition, a study found that CD $8+T$ cells activated by immunotherapy enhance ferroptosis-specific lipid peroxidation in tumor cells, and the increase in ferroptosis also contributes to the antitumor efficacy of immunotherapy[24].

In this study, we used high-throughput methodology technology to comprehensively analyze the genome of HCC and thousands of molecular targets, identify iron metabolism and ferroptosis-related genes closely associated with the prognosis of HCC, construct predictive models for the diagnosis and prognosis of HCC, and explore the relationship with immune infiltration in HCC. Our findings may help improve the early diagnosis rate of HCC and further improve the clinical outcomes of patients under personalized treatment.

\section{Methods}

\section{Acquisition of ferroptosis- and iron metabolism-related genes associated with HCC}

Ferroptosis-related genes were obtained in the ferroptosis pathway (map04216) from the KEGG PATHWAY Database (https://www.genome.jp/kegg/pathway.html). Genes related to iron metabolism were obtained in the pathway of iron uptake and transport (R-HSA-917937) from the Reactome Pathway Database (https://reactome.org/) and cellular iron ion homeostasis (GO:0006879) from the AmiGo2 database (http://amigo.geneontology.org/amigo)[25]. We searched and comprehensively analyzed the 


\section{Identifying differentially expressed genes (DEGs) between $\mathrm{HCC}$ and adjacent nontumor tissues}

The mRNA-sequencing data of patients with $\mathrm{HCC}$ with clinical information were downloaded from the TCGA database (including $370 \mathrm{HCC}$ tissue samples and 50 normal tissue samples for a total of 10,000 encoding mRNA sequences) and the ICGC database (including 202 normal samples and 243 HCC samples for a total of 19,677 encoding mRNA sequences). Matching the mRNA-sequencing data with ferroptosis- and iron metabolism-related genes and using limma, an R package, with an absolute log2fold change $(F C)>1$ and an adjusted $P$ value $<0.05$ to perform differential expression analysis, DEGs related to ferroptosis and iron metabolism were thereby identified. Since the data of the TCGA database and the ICGC database are open to the public and can be downloaded freely, and this study strictly followed the publication guidelines and access policies of the databases, ethical review and approval from an Ethics Committee are not required for the study.

\section{Establishment and validation of a prognostic predictive signature}

Using univariate Cox regression analysis to screen out genes related to OS in patients with HCC, genes with a $P$ value $<0.05$ were considered statistically significant and incorporated into the subsequent LASSO Cox regression. In the LASSO-penalized Cox regression analysis, we adjusted the L1 penalty parameter via 10-fold cross-validation to narrow the number of genes, and genes that appeared with a repetition frequency greater than 900 times in 1000 substitution samplings were considered to be more closely related to OS. Based on a multivariate Cox regression for these genes, we built a prognostic signature. The prognostic risk score was determined using a linear combination of the regression coefficient $(\beta)$ in a multivariate Cox regression model and the expression levels of the genes. Prognostic index $(\mathrm{PI})=(\beta$ * expression level of $\mathrm{ABCB} 6)+(\beta$ * expression level of FLVCR1 $)+(\beta$ * expression level of SLC48A1 $)+(\beta *$ expression level of SLC7A11). X-tile software was used to determine the optimal cut-off value to divide patients with HCC with survival status into the high-risk and low-risk groups. Kaplan-Meier (K-M) survival curves and time-dependent receptor operating characteristic (ROC) curves were performed to evaluate the predictive performance of the prognostic signature on OS.

\section{Independence of the prognostic signature from traditional clinical characteristics}


Univariate and multivariate Cox regression analyses were performed to confirm whether the prognostic signature was independent of other traditional clinical characteristics (including age, AFP, weight, vascular tumor cell, sex, pathological grade and TNM stage) in predicting OS of patients with HCC. Hazard ratios (HRs) and 95\% confidence intervals (Cls) for each variable were calculated. $P<0.05$ was considered statistically significant.

\section{Construction and evaluation of a predictive nomogram}

We integrated the independent predictive factors identified by multivariate Cox regression and constructed a predictive nomogram and corresponding calibration maps using "rms" R software. Calibration and discrimination were carried out to validate the calibration maps. The consistency index (C Index), which was calculated via a bootstrap method with 1000 resamples, was used to evaluate the prediction accuracy of the nomogram compared to the actual result and to graphically plot the actual observed rate and the predicted rate of the nomogram to evaluate the calibration curves. The closer the calibration curve is to the $45^{\circ}$ line, which represents the best prediction, the better is the prognostic prediction performance of the nomogram. ROC curve analysis was performed to validate the sensitivity and specificity of the nomogram compared to a single independent predictor in predicting OS, and decision curve analysis (DCA) was performed to evaluate the clinical benefit that the nomogram can obtain compared to a single independent prognostic predictor. $\mathrm{P}<0.05$ was considered statistically significant.

\section{Internal and external validation of the expression characteristics of ferroptosis- and iron metabolism-related genes}

Wilcoxon signed rank tests in Prism 7.0 (GraphPad, San Diego, CA, USA) were used to validate the expression characteristics of ferroptosis- and iron metabolism-related genes between $\mathrm{HCC}$ and normal tissues in the HCC cohort from ICGC and GSE6764. $P<0.05$ was considered statistically significant. Regression analysis was performed to explore the correction among the expression profiles of genes. ROC curve analysis was performed to validate the predictive ability of the genes for OS.

\section{Estimation of immune cell type fractions}

The microenvironmental cell counting (MCP-counter) method was used for the assessment of immune infiltration, which can reliably and quantitatively analyze the absolute abundance of ten immune cells and stromal cells in heterogeneous tissue from transcriptome data[26]. The R package "MCP counter" was used to convert the mRNA data to the level of nontumor cell infiltration in the tumor microenvironment. Gene expression profiles were prepared using standard annotation files before 
analysis of the MCP counter. The tumor purity and immune fraction were estimated using the "ESTIMATE" R package. For each sample, the sum of all estimated immune cell type scores is equal to 1.

\section{Cell Culture}

Human HCC cell lines (SK-HEP1 and SMMC-7721) were purchased from American Type Culture Collection (ATCC) (Manassas, VA, USA). The cell lines were cultured in DMEM (Gibco, NYC, USA) supplemented with $10 \%$ heat-inactivated fetal bovine serum (Gibco, NYC, USA) at $37^{\circ} \mathrm{C}$ and maintained in a humidified cell incubator with an atmosphere of $5 \% \mathrm{CO} 2$.

\section{Cell Counting Kit-8 (CCK-8) assay}

SK-HEP1 and SMMC- 7721 cells were plated in a 96-well plate with 3000 cells per well and 5 wells as a set and incubated in a humidified cell incubator with an atmosphere of $5 \% \mathrm{CO} 2$ at $37^{\circ} \mathrm{C}$ for 24 hours. Then, after treating the cells with $0-40 \mu \mathrm{M}$ erastin for 72 hours, CCK -8 reagent was added, and incubation was continued for another 2 hours. The OD was measured at $450 \mathrm{~nm}$ using a microplate reader.

\section{Nude mouse xenograft assay}

Male BALB/c-nude mice aged 4-6 weeks were purchased from Shanghai Slac Laboratory Animal Co. LTD (Shanghai, China) for the construction of HCC xenograft mouse models. After resuspending in PBS, SMMC -7721 cells $\left(6 \times 10^{\wedge} 6 /\right.$ mouse $)$ were injected subcutaneously into the ventral side of nude mice. Nude mice were randomly divided into two groups ( 5 mice/group) and kept in a sterile environment with 12 hours of light/12 hours of darkness per day. One week after implantation, when the subcutaneous tumor was visible to the naked eye (approximately $2 \mathrm{~mm}$ ), mice were treated with $40 \mathrm{mg} / \mathrm{kg}$ erastin (intraperitoneal injection, three times a week) or vehicle control (saline). The tumor volume (TV) was calculated according to the following formula: $\operatorname{TV}(\mathrm{mm} 3)=\mathrm{L} \times \mathrm{W}^{\wedge} 2 \times 0.5$

\section{Quantitative real-time polymerase chain reaction (qRT-PCR)}

Total RNA was extracted from SK-HEP1 cells and SMMC-7721 cells treated with erastin using TRIzol reagent (Invitrogen, Carlsbad, USA) and reverse transcribed using a cDNA reverse transcription kit (TransGen, Guangzhou, China) in accordance with the manufacturer's instructions, and the obtained cDNA was amplified using a SYBR Green PCR kit (TransGen, Guangzhou, China). qRT-PCR was performed to detect expression levels in samples. The primers used for qRT-PCR were purchased from TsingKe (Beijing China). Each experiment was repeated three times. The 2- $\Delta \Delta \mathrm{CT}$ methodology was adopted to calculate the expression of genes. 


\section{Statistical analysis}

Student's two-sided t-tests in Prism 7.0 (GraphPad, San Diego, CA, USA) were used to compare the differences between two groups. The results are presented as the mean \pm standard deviation (SD) of at least five independent experiments. $P<0.05$ was considered statistically significant.

\section{Results}

\section{Identification of DEGs related to ferroptosis and iron metabolism in HCC}

A total of 104 ferroptosis- and iron metabolism-related genes were identified to match the mRNAsequencing data in the TCGA and ICGC databases. Using limma with an absolute log2-fold change (FC)> 1 and an adjusted $P$ value $<0.05$ to perform differential expression analysis, we identified 24 DEGs (17 upregulated and 7 downregulated) in TCGA (Figure 1A and 1C) and 16 DEGs (13 upregulated and 3 downregulated) in ICGC (Figure 1B and 1D) that were related to ferroptosis and iron metabolism in HCC.

\section{Comprehensive analysis of the ferroptosis- and iron metabolism-related genes closely associated with prognosis in HCC}

We performed univariate Cox regression to explore the relationship between the expression of the 24 DEGs obtained from TCGA and prognosis using $371 \mathrm{HCC}$ samples with OS rates and survival status in TCGA. Sixteen DEGs were statistically significant $(P<0.05)$ and considered to be associated with the prognosis of HCC. Then, LASSO Cox regression was applied to these genes. LASSO is a penalized regression method that adjusts the regression coefficient with $L 1$ penalty to reduce the final weight of most potential indicators to zero, thereby decreasing the number of indicators with a final weight of nonzero[27]. Based on the LASSO regression with 10-fold cross-validation, we screened 7 genes with a repetition frequency greater than 900 times in 1000 substitution samplings (Figure 1E-F). Matching the 7 genes with 16 DEGs in ICGC, we finally determined that 4 genes (ABCB6, FLVCR1, SLC48A1 and SLC7A11) were significantly associated with prognosis in HCC.

\section{Building the prognostic signature based on the four ferroptosis- and iron metabolism-related genes and validating its predictive performance}

Based on a multivariate Cox regression of the four genes (ABCB6, FLVCR1, SLC48A1 and SLC7A11), we built a prognostic signature. Prognostic index $(P I)=(\beta$ * expression level of $A B C B 6)+(\beta$ * expression level 
of FLVCR1) + $(\beta$ * expression level of SLC48A1) $+(\beta$ * expression level of SLC7A11). The optimal cut-off value was determined using X-tile software and performed to divide 370 patients with HCC in the HCC cohort from TCGA into the high-risk and low-risk groups. OS was significantly worse in the high-risk groups than that in the low-risk groups $(\mathrm{P}<0.001, \mathrm{HR}=3.70,95 \% \mathrm{Cl}: 2.22-6.25)$ (Figure $2 \mathrm{~A})$. Figure $2 \mathrm{C}$ shows the distribution of risk scores corresponding to gene expression levels. The area under the curve (AUC) in the time-dependent ROC at 0.5, 1, 3 and 5 years reached $0.73,0.77,0.71$ and 0.64 (Figure 2D), indicating great specificity and sensitivity of the prognostic signature in predicting OS. We then used the $243 \mathrm{HCC}$ samples in the ICGC to validate the predictive performance of the prognostic signature. PI was calculated according to the formula mentioned earlier, and $243 \mathrm{HCC}$ samples were divided into the highrisk group and low-risk group based on the optimal cutoff value determined by X-tile software. Consistent with the above results, patients with HCC in the high-risk group had a significantly lower OS than those in the low-risk group $(\mathrm{P}<0.001, \mathrm{HR}=2.70,95 \% \mathrm{Cl}$ : 1.49-5.00) (Figure $2 \mathrm{~B})$. The risk score distribution and gene expression are shown in Figure 2E. The AUCs for 0.5-, 1-, 3- and 5-year OS were 0.72, 0.67, 0.73 and 0.62 , respectively (Figure $2 \mathrm{~F}$ ).

\section{Construction and validation of the predictive nomogram in the HCC cohort from TCGA}

To determine whether the predictive ability of the prognostic signature in predicting OS was independent of other traditional clinical characteristics (including age, AFP, weight, vascular tumor cell, sex, pathological grade and TNM stage), we performed univariate and multivariate Cox regression analyses on these variables using $370 \mathrm{HCC}$ samples with clinical information in TCGA. The results determined that TNM stage $(H R=2.038)$ and risk score of the prognostic signature $(H R=1.258)$ were independent predictive factors for predicting OS (Figure $3 \mathrm{~A}$ ). Based on the two independent predictive factors, we constructed a predictive nomogram to quantify the prediction results of individual survival probability at 1,3 and 5 years (Figure 3B). The $C$ index for the nomogram was 0.66 , with 1000 cycles of bootstrapping (95\% Cl: 0.55-0.72), and the calibration curves of the nomogram showed great consistency between the predicted OS rates and actual observations at 1, 3 and 5 years (Figure 3C-E).

We then performed ROC curve analysis to validate the predictive value of the nomogram. The AUCs for 1-, 3 - and 5-year OS with the nomogram were $0.644,0.694$ and 0.667 , respectively, superior to a single independent predictive factor (Figure 3F-H). To further determine the value of the nomogram in clinical decision making, we performed DCA. DCA is a new reliable evaluation tool that quantifies the clinical value of a nomogram by analyzing the clinical results obtained from the decision based on the nomogram and has important value in determining the diagnosis and adjusting the prognosis strategy[28]. We found that compared to a single independent predictive factor, the nomogram could obtain the optimal net benefit at 1, 3 and 5 years (Figure $3 \mathrm{I}-\mathrm{J}$ ). 


\section{The diagnostic models were established and validated for high specificity and sensitivity}

A diagnostic model integrating the four genes was established to distinguish HCC from normal subjects using a stepwise logistic regression method. Diagnostic scores were identified as follows: logit $(P=H C C)$ $=-15.2439+(-0.0327 \times$ ABCB6 expression level $)+(8.0880 \times$ FLVCR1 expression level $)+(3.1229 \times$ SLC48A1 expression level $)+(0.1703 \times$ SLC7A11 expression level). Applying the diagnostic model, there was $92.00 \%$ sensitivity and $98.00 \%$ specificity in the HCC cohort from TCGA (containing 50 normal samples and paired $50 \mathrm{HCC}$ samples) (Figure $4 \mathrm{~A}$ ) and $88.07 \%$ sensitivity and $92.08 \%$ specificity in the HCC cohort from ICGC (containing 202 normal samples and $243 \mathrm{HCC}$ samples) (Figure 4B). ROCs in the HCC cohort from TCGA (AUC $=0.980)($ Figure $4 C)$ and ICGC $(A U C=0.956)$ (Figure 4D) were also determined to have great value in accurately distinguishing HCC from normal samples. Unsupervised hierarchical clustering of the four genes indicated a superior ability to differentiate HCC from normal samples (Figure 4E and 4F).

Since nodules less than $2 \mathrm{~cm}$ in the liver were difficult to distinguish from HCC through radiological or pathological examinations[29], we also constructed a diagnostic model based on the four genes in the training cohort (GSE6764) (containing $35 \mathrm{HCC}$ samples and 17 dysplastic nodule samples) for differentiating nodules from HCC samples and validated it in the test cohort (GSE98620) (containing 49 HCC samples and 24 dysplastic nodule samples). Diagnostic scores were identified as follows: logit $(\mathrm{P}=$ $\mathrm{HCC})=-13.9106+(1.3676 \times \mathrm{ABCB} 6$ expression level $)+(-0.1018 \times$ FLVCR1 expression level $)+(-0.2817$ $\times$ SLC48A1 expression level $)+(1.1909 \times$ SLC7A11 expression level $)$. The AUCs for the diagnostic model reached 0.973 in the training cohort, with $97.14 \%$ sensitivity and $94.12 \%$ specificity (Figure $5 \mathrm{~A}$ and $5 \mathrm{C}$ ), and 0.786 in the test cohort, with $79.59 \%$ sensitivity and $54.17 \%$ specificity (Figure $5 B$ and $5 \mathrm{D}$ ). Figures $5 \mathrm{E}$ and $5 \mathrm{~F}$ show unsupervised hierarchical clustering of the four genes.

\section{Comparison of the immune microenvironment of patients with HCC between the high-risk and low-risk groups}

Since drugs targeting immune checkpoints have been shown to achieve antitumor effects by reversing the immunosuppressive effects of tumors, the expression of immune checkpoints has attracted widespread attention as a biomarker for identifying patients with HCC to receive immunotherapy[30]. The TMB can be used to predict the efficacy of immune checkpoint blockade and has been proven to be a biomarker for identifying patients who can benefit from immunotherapy in several cancer types[31]. In this study, we analyzed the association between risk scores and TMB. Figures $6 \mathrm{~A}$ and $6 \mathrm{~B}$ indicate the differences in TMB in somatic cells in patients with HCC between the high- and low-risk groups. Patients in the high-risk group had a higher TMB than patients in the low-risk group (Figure 6C). A higher OS rate was obtained in patients with low risk and low TMB group than that in patients with high risk and high TMB group $(P<0.0001)$ (Figure 6D). 
The differences in immune infiltration of 22 immune cell types obtained from 289 patients with HCC from the TCGA database are shown in Figure 7A, which may represent an intrinsic feature that can characterize individual differences. Patients with HCC in the high-risk group had higher ratios of M0 macrophages, follicular helper T cells, memory B cells, and neutrophils than those in the low-risk group ( $P$ $<0.05$ ) (Figures 7C-F). Figure 7B shows the relationship between the risk score and the expression of immune checkpoints. We found that the expression levels of CD83, B7H3, OX40 and OX40L in the highrisk group were significantly higher than those in the low-risk group $(P<0.05)$ (Figure $7 G-J)$, suggesting that the poor prognosis of high-risk patients was partly due to the immunosuppressive microenvironment. The results above indicated that abnormal immune infiltration and expression differences of immune checkpoints in $\mathrm{HCC}$ can be used as prognostic indicators and targets for immunotherapy, with important clinical significance.

\section{Internal and external validation of the expression patterns and prognostic predictive performance of the four ferroptosis- and iron metabolism-related genes}

The expression levels of ABCB6, FLVCR1, SLC48A1, and SLC7A11 were significantly higher in the HCC cohort from ICGC than in normal samples $(P<0.001)$ (Figure 8A-D), which was consistent with the predictive analysis of diagnosis and prognosis, demonstrating that the four genes were suitable for constructing diagnostic and prognostic models. For further validation, we detected the expression characteristics of the four genes in the GSE6764 cohort. The four genes presented markedly higher expression in HCC than in dysplastic nodule samples, consistent with the findings above (Figure 8E-H). In addition, the expression profiles of the four genes in multiple cell lines are shown in Figure 8I-L.

Since the four genes exhibited high expression in the tumor tissues, we explored the correlation among the genes. The expression of ABCB6 had synergy with the expression of FLVCR1, as well as the expression of ABCB6 and SLC7A11, ABCB6 and SLC48A1, and SLC48A1 and SLC7A11, which also had the same positive correlation (Figure 9A-D). The correlation between the expression of the four genes by $\mathrm{HCC}$ cells and the immune infiltrate is shown in Figure $9 \mathrm{E}-\mathrm{H}$. K-M curve analysis was performed to validate the predictive value of the four genes in OS. Genes with high expression had lower OS rates than those with low expression (Figure 9I-L). ROCs validated the predictive performance with high sensitivity and specificity (Figure 9M-P).

\section{Inhibition of erastin on the proliferation and progression of $\mathrm{HCC}$ and its possible molecular mechanism}

As an inducer of ferroptosis, erastin was used to evaluate its influence on the development and progression of HCC[32].The chemical formula of erastin was showed in Figure 10A. Performing the CCK8 assay, we found that erastin inhibited cell proliferation in a dose-dependent manner (Figure 10B-C). 
Figure 10D-G indicates that erastin inhibited tumor growth in mice. Lower expression levels of Ki67 and $\mathrm{N}$-cadherin were exhibited in tumor tissues under erastin treatment (Figure $10 \mathrm{H})$.

As it was determined that erastin inhibited the proliferation and progression of HCC, we explored the possible molecular mechanism by which erastin achieves antitumor effects. In the Cancer Therapeutics Response Portal (CTRP) database (http://portals.broadinstitute.org/ctrp/), 52 genes were shown to be regulated by erastin, and their association is exhibited in Figure 11A. By performing Gene Ontology (GO) (Figure 11B) and Kyoto Encyclopedia of Genes and Genomes (KEGG) pathway enrichment analyses (Figure 11C) on these genes, we found that erastin could cause changes in signaling cascades, including Th17 cell differentiation and the IL-17 signaling pathway $(P<0.05)$. This result indicated that the IL-17 signaling pathway is a potential target affected by erastin in this study.

\section{Discussion}

As a major leading cause of cancer-related mortality worldwide, HCC presents a major health burden for society[33]. Among the current multiple treatments, liver transplantation and tumor ablation are still the only options that may lead to a cure[34]. However, most patients are diagnosed at an advanced stage, and these treatments cannot be selected. The 5-year recurrence rate is very high even in patients who have received liver resection or liver transplantation[35; 36], and the 5-year survival rate is still low[37]. Since HCC is a molecular heterogeneous malignant tumor, its molecular characteristics are related to corresponding biological behaviors, including cell regeneration, microvascular invasion, and distant metastasis[38], and play an important role in the prognosis of HCC. Therefore, it is necessary to identify key molecular markers that affect the prognosis of HCC, thereby optimizing the early diagnosis of HCC and strengthening treatment to improve the clinical outcome of HCC.

The development of high-throughput array technology provides an opportunity to explore novel genes involved in the occurrence and progression of $\mathrm{HCC}[39]$. Ferroptosis is a regulated autophagic cell death process in which iron-dependent oxidation plays a key role[40]. Disturbances in iron metabolism cause excessive intracellular iron storage and may induce ferroptosis[41]. Ferroptosis is regulated by several genes[42]. Previous studies have confirmed that ferroptosis is an effective mechanism for inducing HCC cell death, but its specific molecular changes and mechanism of action are not fully understood[43; 44]. In this study, we aimed to analyze HCC-related RNA sequences obtained through high-throughput array technology using Cox proportional hazards regression and LASSO methods to determine ferroptosis- and iron metabolism-related genes that were associated with the prognosis of HCC. We found that the prognosis model constructed by four genes (ABCB6, FLVCR1, SLC48A1 and SLC7A11) independently predicted the prognosis of patients with HCC with superior prediction performance. ABCB6 belongs to the $B$ subfamily of $A B C$ transporters, which is a porphyrin energy-dependent transporter[45]. Previous studies have reported a correlation between hepatitis $C$ virus-associated hepatocellular carcinoma and increased ABCB6 mRNA levels. ABCB6 mRNA and DNA methylation levels help predict early intrahepatic recurrence[46]. Feline leukemia virus subgroup $C$ receptor 1, encoded by FLVCR1, plays an important role in iron metabolism, participating in the outflow of iron metabolism, preventing oxidative damage caused 
by excessive iron[47]. Studies have found that FLVCR1 expression is correlated with the prognosis of HCC[48]. SLC48A1 is an endosomal heme transporter that participates in the process of heme iron transport in iron metabolism[49]. Lipid peroxide is triggered by lipid peroxidation, and this process is strictly regulated by SLC7A11 (a key component of the cystine-glutamate antiporter); when lipid peroxide is excessively accumulated, ferroptosis can be induced[50; 51]. It was reported that the expression of SLC7A11 is related to the prognosis of HCC[52]. In the study, the corresponding nomogram also helps clinicians make better clinical decisions and develop treatment strategies by the four-gene model. The diagnostic models based on the four genes were useful for the early diagnosis of HCC with high specificity and sensitivity.

Immunotherapy is a tumor treatment method that uses the body's own immune system to produce an antitumor response[53]. In order to avoid the antitumor immune response during the development of many types of tumors, immunosuppressive mechanisms will be initiated, and with increased immunosuppressive cells and immunosuppressive molecules, low-immunogenic cancer cells will be selected and an immunosuppressive network (immune escape) will be established[54]. By blocking immunosuppressive mechanisms and the function of immunosuppressive cells, potential antitumor immune responses can be triggered[55]. In recent years, manipulation of immune checkpoints or pathways has become an important and effective form of immunotherapy[53], and high TMB has been identified to correlate with good outcomes of immune checkpoint inhibitor treatments[56]. In this study, we found that patients with $\mathrm{HCC}$ with high risk scores identified by the ferroptosis and iron metabolism signatures had higher TMB levels and higher proportions of M0 macrophages, follicular helper T cells, memory B cells and neutrophils, which may indicate that the poor prognosis in the high-risk group may be due to a stronger immunosuppressive effect. When detecting immune checkpoints, higher expression of $\mathrm{CD} 83, \mathrm{~B} 7 \mathrm{H} 3, \mathrm{OX} 40$ and OX40L was exhibited in the high-risk group. These differences promote the growth and progression of HCC, leading to a poor prognosis for HCC. In addition, the findings above suggest that patients in the high-risk group may benefit more from immune checkpoint inhibitor therapy than patients in the low-risk group. This provides new insight for tumor immunotherapy.

Erastin has been determined to be an inducer of ferroptosis in previous studies and has been identified as an inhibitor of cystine/glutamate antiporter (xCT) and glutathione synthesis[57; 58]. In this study, we found that erastin has an antitumor effect by inhibiting the proliferation and progression of HCC cells. Erastin treatment has been shown to inhibit tumor growth in mouse tumor models, which provides new ideas for the treatment of HCC. In addition, erastin could change TH17 cell differentiation and the IL-17 signaling pathway by bioinformatics analysis. IL -17 is a universal cytokine in the tumor microenvironment. In existing tumors, $\mathrm{IL}-17$ achieves an antitumor effect by activating immune cells and inducing indirect immunity[58; 59]. The regulatory potential of the IL-17 immune axis makes IL-17 a compelling target in cancer immunotherapy. These results suggest that the ferroptosis inducer erastin may be regarded as a potential agent of cancer immunotherapy.

\section{Conclusion}


In summary, we identified four ferroptosis- and iron metabolism-related genes with great predictive value in the OS of HCC, and the prognostic and diagnostic models based on the four genes indicated superior diagnostic and predictive performance. As an inducer of ferroptosis, erastin showed an antitumor effect by inhibiting the proliferation and progression of HCC. Through bioinformatics analysis, erastin was shown to affect $\mathrm{TH} 17$ cell differentiation and the IL -17 signaling pathway, indicating that it is a potential targeted drug for immunotherapy.

\section{Abbreviations}

HCC: Hepatocellular carcinoma

LASSO: The least absolute shrinkage and selection operator

OS: Overall survival

$T M B$ : Tumor mutation burden

$D E G$ : Differentially expressed gene

HR: Hazard ratio

Cl: Confidence intervals

TCGA: The Cancer Genome Atlas

ICGC: International Cancer Genome Consortium

KEGG: Kyoto Encyclopedia of Genes and Genomes

GO: Gene Ontology

ABCB6: ATP binding cassette subfamily B member 6

FLVCR1: FLVCR heme transporter 1

SLC48A1: Solute carrier family 48 member 1

SLC7A11: solute carrier family 7 member 11

ROC: Receiver operator characteristic curve

AUC: Area Under Curve

$D C A$ : Decision Curve Analysis

CCK -8 assay: Cell Counting Kit-8 Assay 


\section{Declarations}

\section{Data and materials Availability}

The data and materials used to support the findings of this study are available from the corresponding author upon request.

\section{Acknowledgments}

The authors of the present work are truly grateful to the ICGC, GEO and TCGA databases for the availability of the data.

\section{Funding}

This study was supported by National Natural Science Foundation of China (Nos. 81803778), and The Key Research and development Project of Zhejiang Province (No. 2018C03024), and The Public Welfare Research Program of Zhejiang Province (Nos. LQ20H160056 and LGD19h160002), The Natural Science Foundation of Zhejiang Province (No. LYQ20H280003).

\section{Author information}

\section{Affiliations}

Key Laboratory of Imaging Diagnosis and Minimally Invasive Intervention Research, Lishui Hospital, School of Medicine, Zhejiang University, Lishui 323000, China

Bufu Tang, Jinyu Zhu, Jie Li, Kai Fan

Department of Radiology, the Fifth Affiliated Hospital of Wenzhou Medical University, Lishui 323000, China

Yang Gao, Shimiao Cheng, Chunli Kong, Liyun Zheng, Fazong Wu, Qiaoyou Weng, Chenying Lu1, Jiansong Ji

\section{Contributions}

Jiansong Ji and Chenying Lu conceived and designed the experiments. Bufu Tang and Jinyu Zhu performed the experiments. Jie Li, Kai Fan, Yang Gao and Shimiao Cheng analyzed the data. Chunli Kong, Liyun Zheng, Fazong Wu and Qiaoyou Weng contributed analysis tools. Bufu Tang and Jinyu Zhu wrote the paper. Jiansong Ji and Chenying Lu edited the paper. All authors read and approved the final manuscript.

\section{Corresponding author}


Correspondence to Jiansong Ji and Chenying Lu,

\section{Ethics declarations}

\section{Ethics approval}

The mRNA-sequencing data and corresponding clinical information in our study were obtained from TCGA and ICGC databases and were freely available to the public, and this study also strictly followed the publication guidelines and access policies of these databases, this study does not require ethical review and approval from an Ethics Committee.

\section{Consent for publication}

Not applicable.

\section{Conflicts of Interest}

The authors declare no conflict of interest.

\section{References}

[1] S. Stuver, D. Trichopoulos, H.O. Adami, D. Hunter, and D. Trichopoulos, Cancer of the liver and biliary tract. (2008).

[2] F. Bray, J. Ferlay, I. Soerjomataram, R.L. Siegel, L.A. Torre, and A. Jemal, Global cancer statistics 2018: GLOBOCAN estimates of incidence and mortality worldwide for 36 cancers in 185 countries. CA: a cancer journal for clinicians 68 (2018) 394-424.

[3] K. Popat, K. Mcqueen, and T.W.J.B.P.R.C.A. Feeley, The global burden of cancer. 27 (2013) 399-408.

[4] M. Minagawa, I. Ikai, Y. Matsuyama, Y. Yamaoka, and M. Makuuchi, Staging of hepatocellular carcinoma: assessment of the Japanese TNM and AJCC/UICC TNM systems in a cohort of 13,772 patients in Japan. Annals of surgery 245 (2007) 909-22.

[5] J.U. Marquardt, P.R. Galle, and A. Teufel, Molecular diagnosis and therapy of hepatocellular carcinoma (HCC): an emerging field for advanced technologies. Journal of hepatology 56 (2012) 267-75.

[6] T. Couri, and A. Pillai, Goals and targets for personalized therapy for HCC. Hepatology international 13 (2019) 125-137.

[7] T. El Jabbour, S.M. Lagana, and H. Lee, Update on hepatocellular carcinoma: Pathologists' review. World journal of gastroenterology 25 (2019) 1653-1665.

[8] N.C. Andrews, Disorders of iron metabolism. The New England journal of medicine 341 (1999) 198695. 
[9] N.C. Andrews, Forging a field: the golden age of iron biology. Blood 112 (2008) 219-30.

[10] D.H. Manz, N.L. Blanchette, B.T. Paul, F.M. Torti, and S.V. Torti, Iron and cancer: recent insights. Annals of the New York Academy of Sciences 1368 (2016) 149-61.

[11] L. Zhou, B. Zhao, L. Zhang, S. Wang, D. Dong, H. Lv, and P. Shang, Alterations in Cellular Iron Metabolism Provide More Therapeutic Opportunities for Cancer. International journal of molecular sciences 19 (2018).

[12] M.K. Ali, R.Y. Kim, R. Karim, J.R. Mayall, K.L. Martin, A. Shahandeh, F. Abbasian, M.R. Starkey, V. Loustaud-Ratti, D. Johnstone, E.A. Milward, P.M. Hansbro, and J.C. Horvat, Role of iron in the pathogenesis of respiratory disease. The international journal of biochemistry \& cell biology 88 (2017) 181-195.

[13] A. Fonseca-Nunes, P. Jakszyn, and A. Agudo, Iron and cancer risk-a systematic review and metaanalysis of the epidemiological evidence. Cancer epidemiology, biomarkers \& prevention : a publication of the American Association for Cancer Research, cosponsored by the American Society of Preventive Oncology 23 (2014) 12-31.

[14] Y. Chen, Z. Fan, Y. Yang, and C. Gu, Iron metabolism and its contribution to cancer (Review). International journal of oncology 54 (2019) 1143-1154.

[15] B.R. Stockwell, J.P. Friedmann Angeli, H. Bayir, A.I. Bush, M. Conrad, S.J. Dixon, S. Fulda, S. Gascon, S.K. Hatzios, V.E. Kagan, K. Noel, X. Jiang, A. Linkermann, M.E. Murphy, M. Overholtzer, A. Oyagi, G.C. Pagnussat, J. Park, Q. Ran, C.S. Rosenfeld, K. Salnikow, D. Tang, F.M. Torti, S.V. Torti, S. Toyokuni, K.A. Woerpel, and D.D. Zhang, Ferroptosis: A Regulated Cell Death Nexus Linking Metabolism, Redox Biology, and Disease. Cell 171 (2017) 273-285.

[16] E. Ooko, M.E. Saeed, O. Kadioglu, S. Sarvi, M. Colak, K. Elmasaoudi, R. Janah, H.J. Greten, and T. Efferth, Artemisinin derivatives induce iron-dependent cell death (ferroptosis) in tumor cells. Phytomedicine : international journal of phytotherapy and phytopharmacology 22 (2015) 1045-54.

[17] H. Yamaguchi, J.L. Hsu, C.T. Chen, Y.N. Wang, M.C. Hsu, S.S. Chang, Y. Du, H.W. Ko, R. Herbst, and M.C. Hung, Caspase-independent cell death is involved in the negative effect of EGF receptor inhibitors on cisplatin in non-small cell lung cancer cells. Clinical cancer research : an official journal of the American Association for Cancer Research 19 (2013) 845-54.

[18] A. Houessinon, C. Francois, C. Sauzay, C. Louandre, G. Mongelard, C. Godin, S. Bodeau, S. Takahashi, Z. Saidak, L. Gutierrez, J.M. Regimbeau, N. Barget, J.C. Barbare, N. Ganne, B. Chauffert, R. Coriat, and A. Galmiche, Metallothionein-1 as a biomarker of altered redox metabolism in hepatocellular carcinoma cells exposed to sorafenib. Molecular cancer 15 (2016) 38. 
[19] J. Nie, B. Lin, M. Zhou, L. Wu, and T. Zheng, Role of ferroptosis in hepatocellular carcinoma. Journal of cancer research and clinical oncology 144 (2018) 2329-2337.

[20] L.B. Alexandrov, S. Nik-Zainal, D.C. Wedge, S.A. Aparicio, S. Behjati, A.V. Biankin, G.R. Bignell, N. Bolli, A. Borg, A.L. Borresen-Dale, S. Boyault, B. Burkhardt, A.P. Butler, C. Caldas, H.R. Davies, C. Desmedt, R. Eils, J.E. Eyfjord, J.A. Foekens, M. Greaves, F. Hosoda, B. Hutter, T. Ilicic, S. Imbeaud, M. Imielinski, N. Jager, D.T. Jones, D. Jones, S. Knappskog, M. Kool, S.R. Lakhani, C. Lopez-Otin, S. Martin, N.C. Munshi, H. Nakamura, P.A. Northcott, M. Pajic, E. Papaemmanuil, A. Paradiso, J.V. Pearson, X.S. Puente, K. Raine, M. Ramakrishna, A.L. Richardson, J. Richter, P. Rosenstiel, M. Schlesner, T.N. Schumacher, P.N. Span, J.W. Teague, Y. Totoki, A.N. Tutt, R. Valdes-Mas, M.M. van Buuren, L. van 't Veer, A. Vincent-Salomon, N. Waddell, L.R. Yates, J. Zucman-Rossi, P.A. Futreal, U. McDermott, P. Lichter, M. Meyerson, S.M. Grimmond, R. Siebert, E. Campo, T. Shibata, S.M. Pfister, P.J. Campbell, and M.R. Stratton, Signatures of mutational processes in human cancer. Nature 500 (2013) 415-21.

[21] Ton N., Schumacher, Can, Kesmir, Marit M., and van Buuren, Biomarkers in Cancer Immunotherapy.

[22] Z.R. Chalmers, C.F. Connelly, D. Fabrizio, L. Gay, S.M. Ali, R. Ennis, A. Schrock, B. Campbell, A. Shlien, J. Chmielecki, F. Huang, Y. He, J. Sun, U. Tabori, M. Kennedy, D.S. Lieber, S. Roels, J. White, G.A. Otto, J.S. Ross, L. Garraway, V.A. Miller, P.J. Stephens, and G.M. Frampton, Analysis of 100,000 human cancer genomes reveals the landscape of tumor mutational burden. Genome medicine 9 (2017) 34.

[23] E.M. Van Allen, N. Wagle, P. Stojanov, D.L. Perrin, K. Cibulskis, S. Marlow, J. Jane-Valbuena, D.C. Friedrich, G. Kryukov, S.L. Carter, A. McKenna, A. Sivachenko, M. Rosenberg, A. Kiezun, D. Voet, M. Lawrence, L.T. Lichtenstein, J.G. Gentry, F.W. Huang, J. Fostel, D. Farlow, D. Barbie, L. Gandhi, E.S. Lander, S.W. Gray, S. Joffe, P. Janne, J. Garber, L. MacConaill, N. Lindeman, B. Rollins, P. Kantoff, S.A. Fisher, S. Gabriel, G. Getz, and L.A. Garraway, Whole-exome sequencing and clinical interpretation of formalin-fixed, paraffin-embedded tumor samples to guide precision cancer medicine. Nature medicine 20 (2014) 682-8.

[24] W. Wang, M. Green, J.E. Choi, M. Gijón, P.D. Kennedy, J.K. Johnson, P. Liao, X. Lang, I. Kryczek, and A.J.N. Sell, CD8+ T cells regulate tumour ferroptosis during cancer immunotherapy.

[25] S. Zhang, W. Chang, H. Wu, Y.-H. Wang, Y.-W. Gong, Y.-L. Zhao, L. Shanhui, H.-Z. Wang, R. Svatek, R. Rodriguez, and Z.-P. Wang, Pan-cancer analysis of iron metabolic landscape across the Cancer Genome Atlas: ZHANG et al. Journal of Cellular Physiology 235 (2019).

[26] E. Becht, N.A. Giraldo, L. Lacroix, B. Buttard, N. Elarouci, F. Petitprez, J. Selves, P. Laurent-Puig, C. Sautes-Fridman, W.H. Fridman, and A. de Reynies, Estimating the population abundance of tissueinfiltrating immune and stromal cell populations using gene expression. Genome biology 17 (2016) 218.

[27] T. Park, and G.J.P.o.t.A.S.A. Casella, The Bayesian Lasso. 103 681-686.

[28] A.J. Vickers, A.M. Cronin, E.B. Elkin, and M. Gonen, Extensions to decision curve analysis, a novel method for evaluating diagnostic tests, prediction models and molecular markers. 8 53-0. 
[29] Characterization of small nodules in cirrhosis by assessment of vascularity: The problem of hypovascular hepatocellular carcinoma \%J Hepatology. 42 (2005).

[30] S.L. Topalian, Targeting Immune Checkpoints in Cancer Therapy. Jama 318 (2017) 1647-1648.

[31] T.A. Chan, M. Yarchoan, E. Jaffee, C. Swanton, S.A. Quezada, A. Stenzinger, and S. Peters, Development of tumor mutation burden as an immunotherapy biomarker: utility for the oncology clinic. Annals of oncology : official journal of the European Society for Medical Oncology 30 (2019) 44-56.

[32] Y. Shibata, H. Yasui, K. Higashikawa, N. Miyamoto, and Y. Kuge, Erastin, a ferroptosis-inducing agent, sensitized cancer cells to X-ray irradiation via glutathione starvation in vitro and in vivo. PloS one 14 (2019) e0225931.

[33] K. Sartorius, B. Sartorius, C. Aldous, P.S. Govender, and T.E. Madiba, Global and country underestimation of hepatocellular carcinoma (HCC) in 2012 and its implications. Cancer epidemiology 39 (2015) 284-90.

[34] G. Garcia-Tsao, and J.K. Lim, Management and treatment of patients with cirrhosis and portal hypertension: recommendations from the Department of Veterans Affairs Hepatitis C Resource Center Program and the National Hepatitis C Program. The American journal of gastroenterology 104 (2009) 1802-29.

[35] A. Forner, J.M. Llovet, and J. Bruix, Hepatocellular carcinoma. 10 (2006) 339-351.

[36] T. Reck, F. K?ckerling, A. Altendorf-Hofmann, P. Haller-Pittrow, C. Wittekind, and W.J.E.J.o.C. Hohenberger, Liver resection and liver transplantation for primary hepatocellular carcinoma. 33 S278.

[37] B. Njei, Y. Rotman, I. Ditah, and J.K. Lim, Emerging trends in hepatocellular carcinoma incidence and mortality. Hepatology (Baltimore, Md.) 61 (2015) 191-9.

[38] D.J. Erstad, B.C. Fuchs, and K.K. Tanabe, Molecular signatures in hepatocellular carcinoma: A step toward rationally designed cancer therapy. Cancer 124 (2018) 3084-3104.

[39] M. Nakamura, T. Chiba, K. Kanayama, H. Kanzaki, T. Saito, Y. Kusakabe, and N. Kato, Epigenetic dysregulation in hepatocellular carcinoma: an up-to-date review. Hepatology research : the official journal of the Japan Society of Hepatology 49 (2019) 3-13.

[40] M. Gao, P. Monian, Q. Pan, W. Zhang, J. Xiang, and X. Jiang, Ferroptosis is an autophagic cell death process. Cell research 26 (2016) 1021-32.

[41] A.R. Bogdan, M. Miyazawa, K. Hashimoto, and Y. Tsuji, Regulators of Iron Homeostasis: New Players in Metabolism, Cell Death, and Disease. Trends in biochemical sciences 41 (2016) 274-286. 
[42] H. Yu, P. Guo, X. Xie, Y. Wang, and G. Chen, Ferroptosis, a new form of cell death, and its relationships with tumourous diseases. Journal of cellular and molecular medicine 21 (2017) 648-657.

[43] C. Louandre, Z. Ezzoukhry, C. Godin, J.C. Barbare, J.C. Maziere, B. Chauffert, and A. Galmiche, Irondependent cell death of hepatocellular carcinoma cells exposed to sorafenib. International journal of cancer 133 (2013) 1732-42.

[44] X. Sun, Z. Ou, R. Chen, X. Niu, D. Chen, R. Kang, and D. Tang, Activation of the p62-Keap1-NRF2 pathway protects against ferroptosis in hepatocellular carcinoma cells. Hepatology (Baltimore, Md.) 63 (2016) 173-84.

[45] R.C. Boswell-Casteel, Y. Fukuda, and J.D. Schuetz, ABCB6, an ABC Transporter Impacting Drug Response and Disease. The AAPS journal 20 (2017) 8.

[46] R. Tsunedomi, N. lizuka, K. Yoshimura, M. lida, M. Tsutsui, N. Hashimoto, S. Kanekiyo, K. Sakamoto, T. Tamesa, and M. Oka, ABCB6 mRNA and DNA methylation levels serve as useful biomarkers for prediction of early intrahepatic recurrence of hepatitis $C$ virus-related hepatocellular carcinoma. International journal of oncology 42 (2013) 1551-9.

[47] D. Chiabrando, M. Castori, M. di Rocco, M. Ungelenk, S. Giesselmann, M. Di Capua, A. Madeo, P. Grammatico, S. Bartsch, C.A. Hubner, F. Altruda, L. Silengo, E. Tolosano, and I. Kurth, Mutations in the Heme Exporter FLVCR1 Cause Sensory Neurodegeneration with Loss of Pain Perception. PLoS genetics 12 (2016) e1006461.

[48] Y. Shen, X. Li, B. Zhao, Y. Xue, S. Wang, X. Chen, J. Yang, H. Lv, and P. Shang, Iron metabolism gene expression and prognostic features of hepatocellular carcinoma. Journal of cellular biochemistry 119 (2018) 9178-9204.

[49] A.A. Khan, and J.G. Quigley, Heme and FLVCR-related transporter families SLC48 and SLC49. Molecular aspects of medicine 34 (2013) 669-82.

[50] T. Liu, L. Jiang, O. Tavana, and W. Gu, The Deubiquitylase OTUB1 Mediates Ferroptosis via Stabilization of SLC7A11. Cancer research 79 (2019) 1913-1924.

[51] X. Lang, M.D. Green, W. Wang, J. Yu, J.E. Choi, L. Jiang, P. Liao, J. Zhou, Q. Zhang, A. Dow, A.L. Saripalli, I. Kryczek, S. Wei, W. Szeliga, L. Vatan, E.M. Stone, G. Georgiou, M. Cieslik, D.R. Wahl, M.A. Morgan, A.M. Chinnaiyan, T.S. Lawrence, and W. Zou, Radiotherapy and Immunotherapy Promote Tumoral Lipid Oxidation and Ferroptosis via Synergistic Repression of SLC7A11. Cancer discovery 9 (2019) 1673-1685.

[52] C. Yue, Y. Ren, H. Ge, C. Liang, Y. Xu, G. Li, J.J.O. Wu, and Therapy, Comprehensive analysis of potential prognostic genes for the construction of a competing endogenous RNA regulatory network in hepatocellular carcinoma. Volume 12 561-576. 
[53] G. Abril-Rodriguez, and A. Ribas, SnapShot: Immune Checkpoint Inhibitors. Cancer cell 31 (2017) 848848.e1.

[54] G.P. Dunn, A.T. Bruce, H. Ikeda, L.J. Old, and R.D.J.N.I. Schreiber, Cancer immunoediting: from immunosurveillance to tumor escape. 3 991-998.

[55] Pardoll, and D. M., The blockade of immune checkpoints in cancer immunotherapy. 12 252-264.

[56] F. Galuppini, C.A.D. Pozzo, J. Deckert, F. Loupakis, and R.B.J.C.C. International, Tumor mutation burden: from comprehensive mutational screening to the clinic. 19 (2019).

[57] S.J. Dixon, K.M. Lemberg, M.R. Lamprecht, R. Skouta, E.M. Zaitsev, C.E. Gleason, D.N. Patel, A.J. Bauer, A.M. Cantley, and W.S. Yang, Ferroptosis: An Iron-Dependent Form of Nonapoptotic Cell Death. 149.

[58] S.J. Dixon, P.D. N, W. Matthew, S. Rachid, L.E. D, H. Miki, T.A. G, G.C. E, T.N. P, and S.B.S.J. eLife, Pharmacological inhibition of cystine-glutamate exchange induces endoplasmic reticulum stress and ferroptosis. 3 .

[59] Veldhoen, and M.J.N. Immunology, Interleukin 17 is a chief orchestrator of immunity. 18 612-621.

\section{Figures}

A

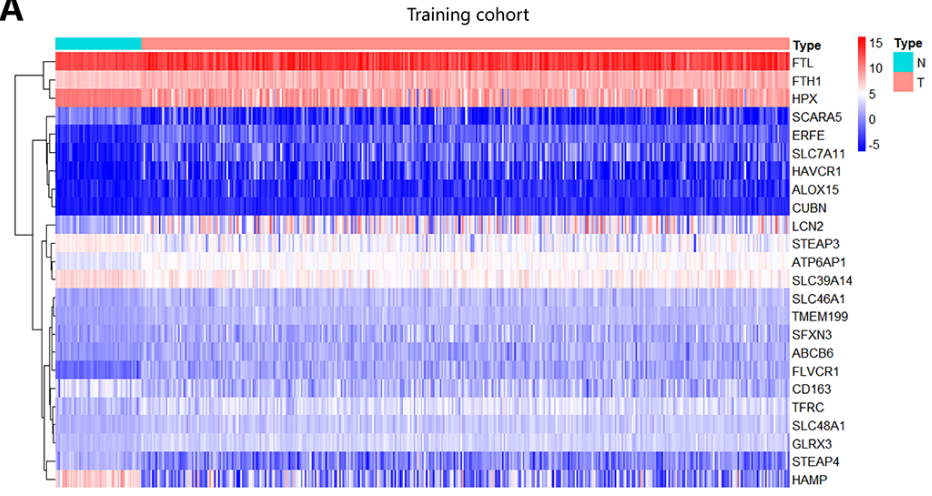

C
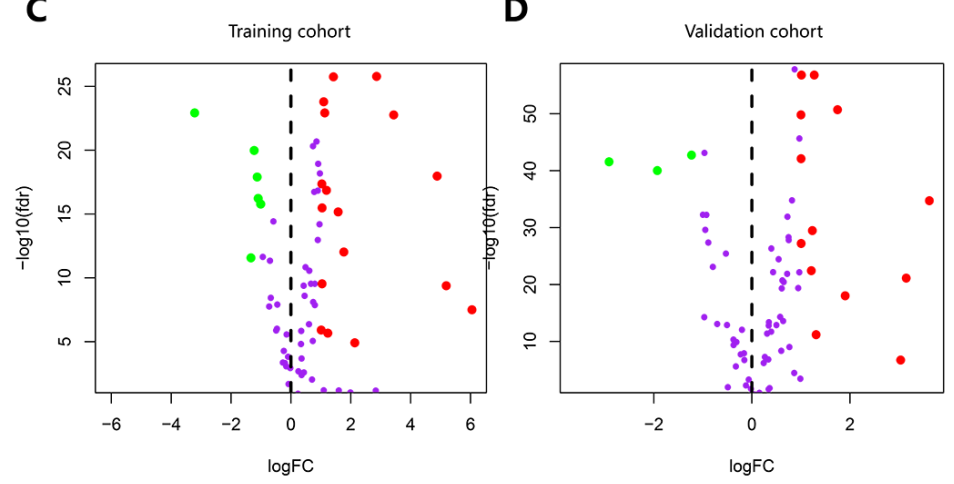

B

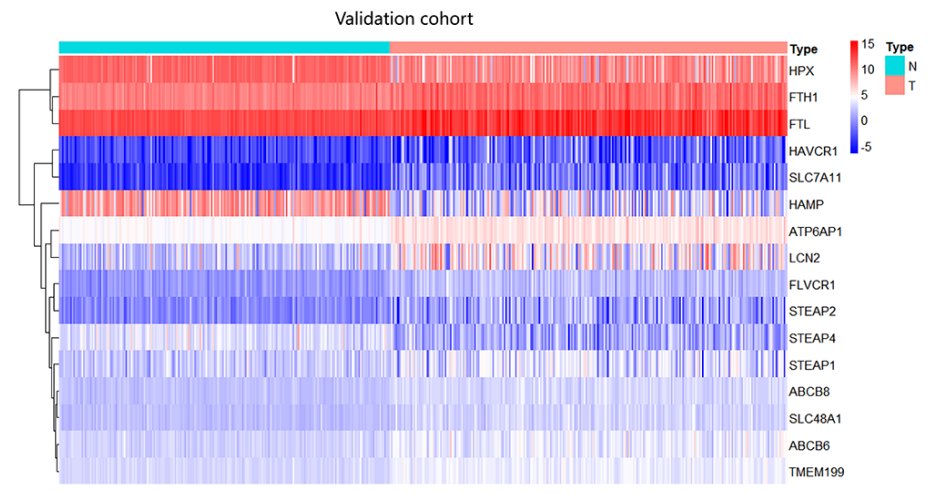

$\mathbf{E}$
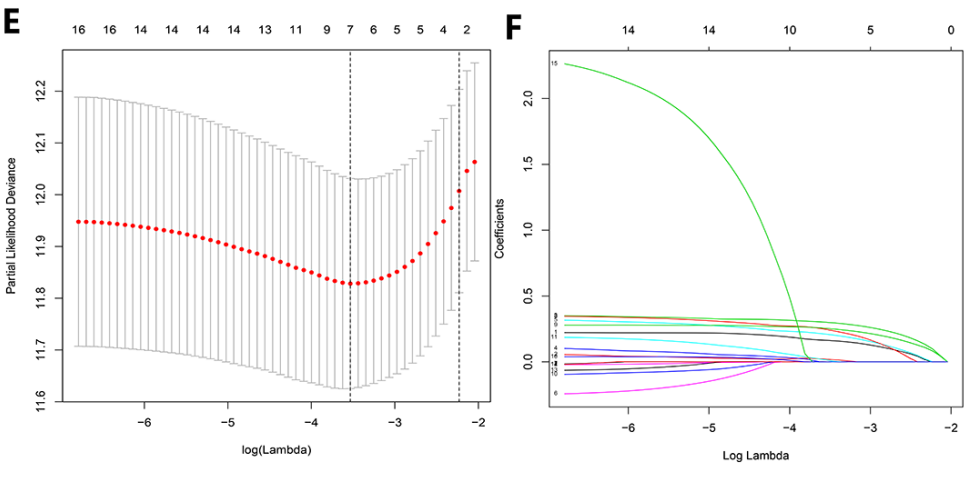

Figure 1 
Heatmap, volcano plot and LASSO Cox regression identified the DEGs closely associated with prognosis in HCC. A and C Gene expression levels in the TCGA database. B and D Gene expression levels in the ICGC database. E and F LASSO Cox regression was performed to identify the DEGs closely related to the prognosis of HCC.

A

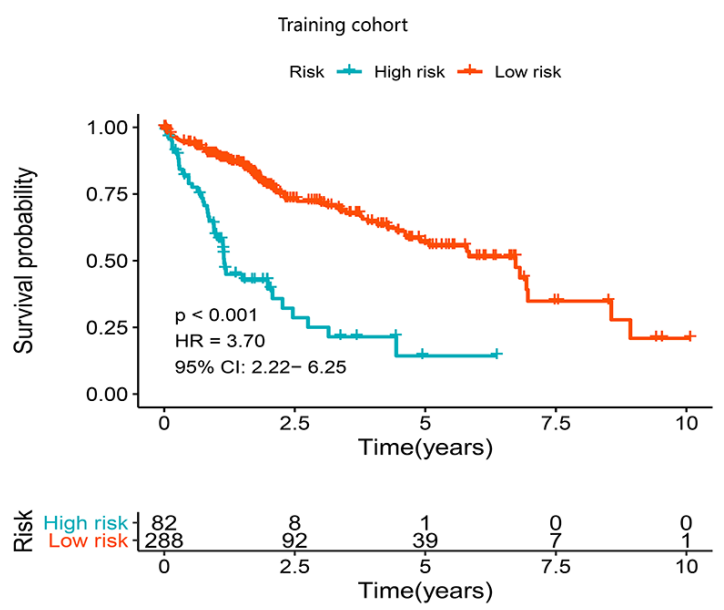

C

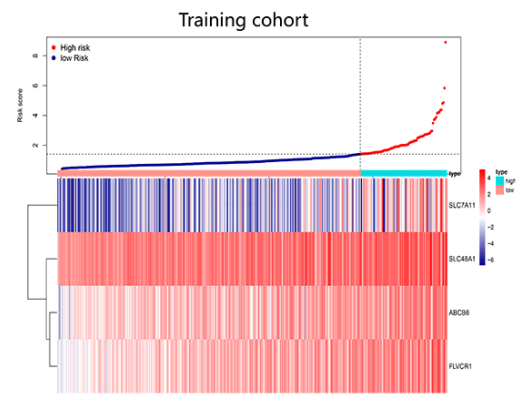

D

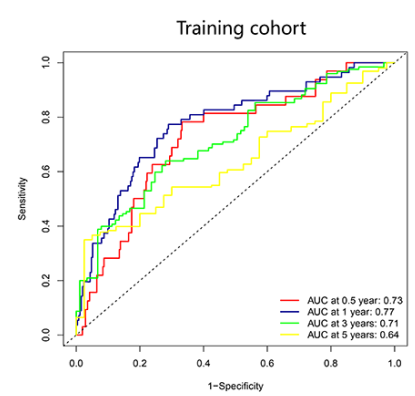

B
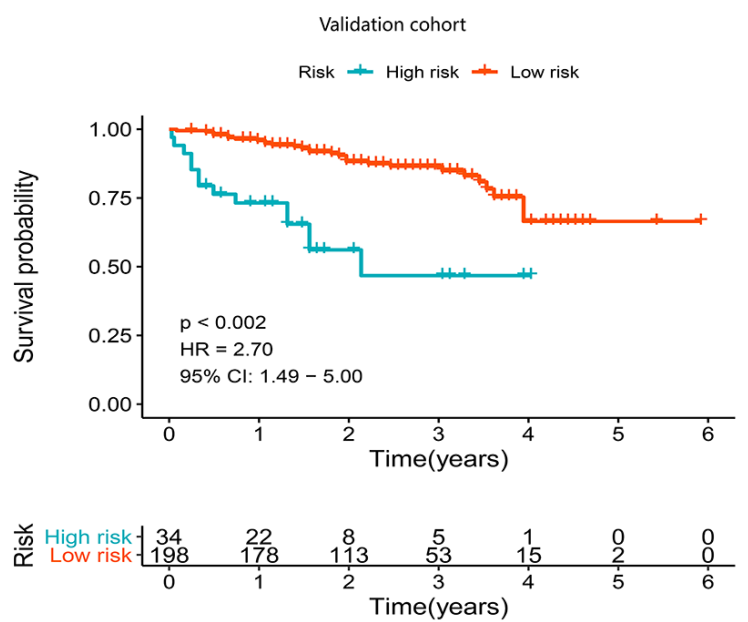

E

$\mathbf{F}$

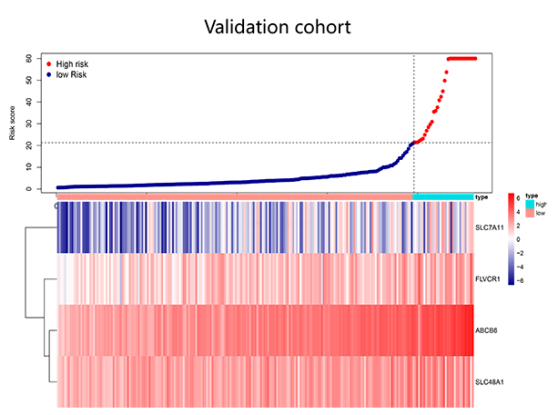

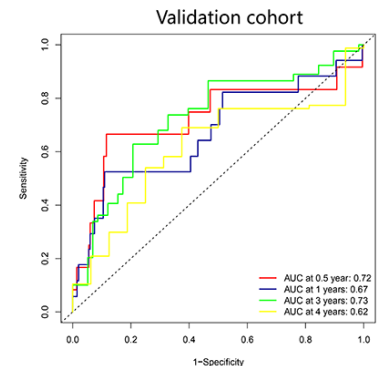

Figure 2

K-M survival analysis, risk score distribution and time-dependent ROC curves of a prognostic model in the HCC cohort from TCGA (A, C-D) and ICGC (B, E-F). A and B K-M survival curves indicated that the OS in the high-risk group was markedly poorer than that in the low-risk group $(P<0.001)$. $C$ and $E$ Distribution of risk scores under different gene expression characteristics in HCC. D and F Time-dependent ROC curve analysis for measuring the predictive performance on OS. 
A

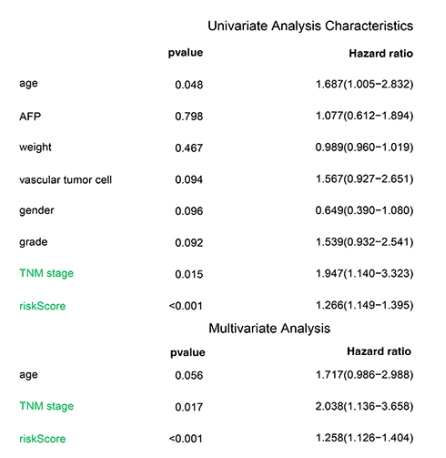

C

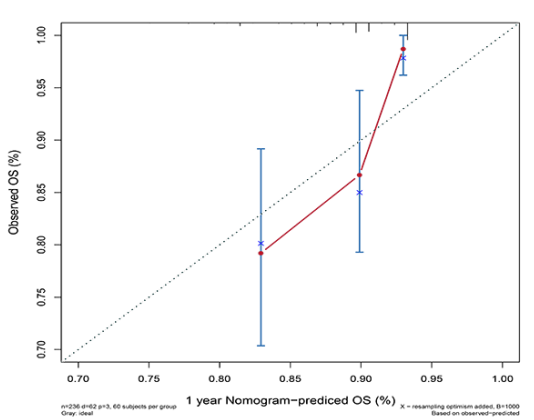

$\mathbf{F}$

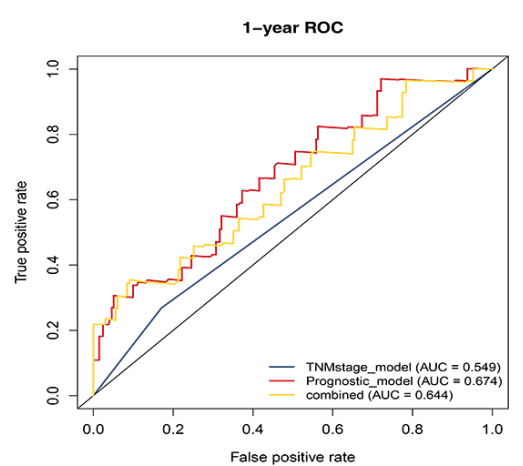

I

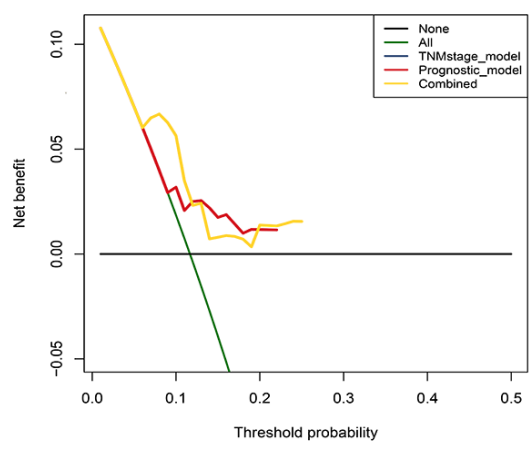

B

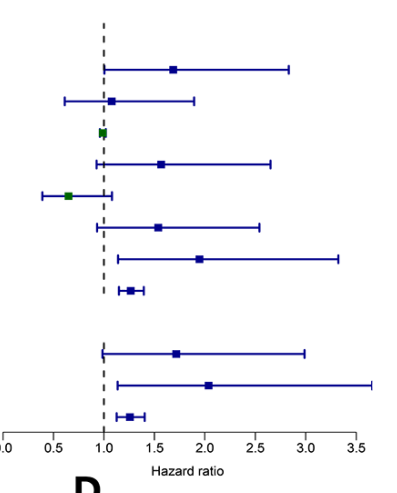

D

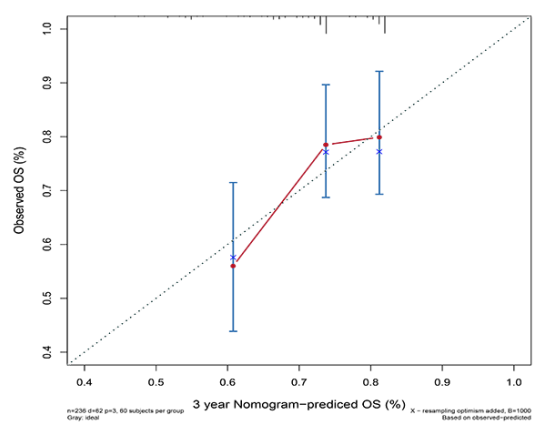

G

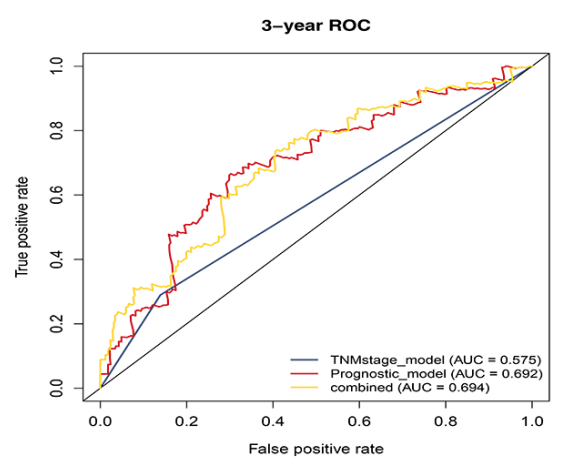

J

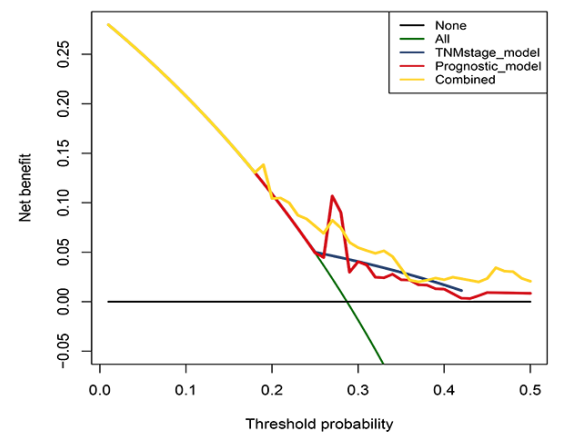

Total Points
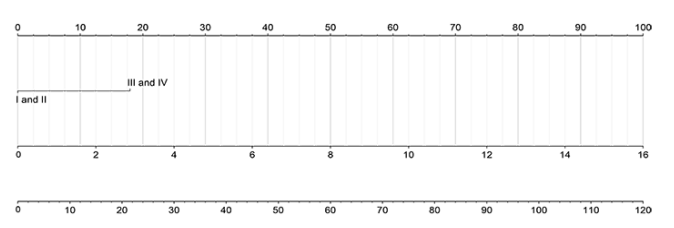

3-year survival Probability

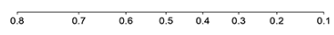

E

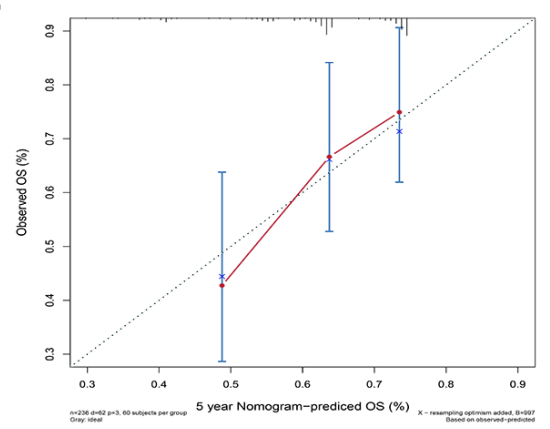

H
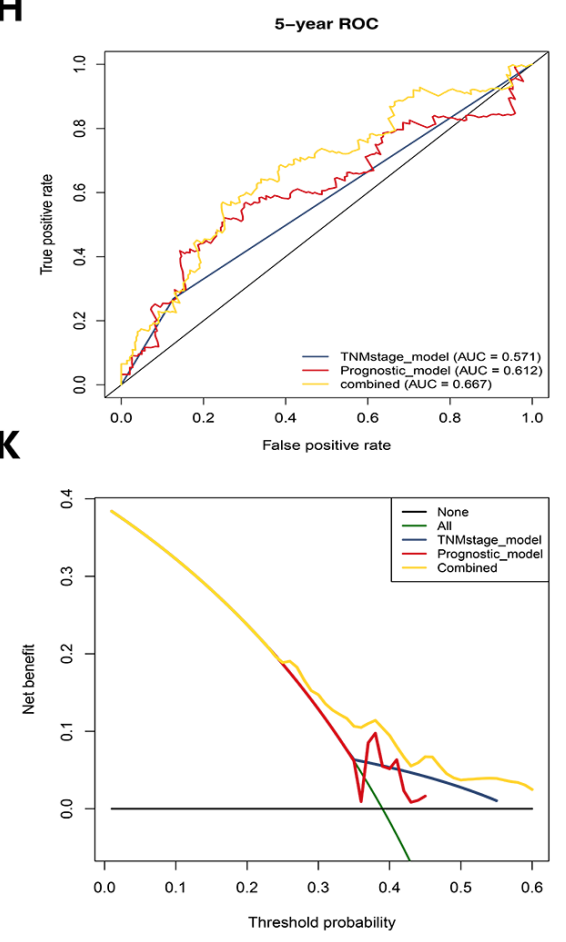

Figure 3

Construction and validation of a predictive nomogram. A Univariate and multivariate Cox regression confirmed that the prognostic signature and TNM stage were independent prognostic predictors. B The nomogram for predicting the OS of patients with HCC at 1, 3, and 5 years. C-E Calibration curves of the nomogram for OS prediction at 1, 3, and 5 years. F-H ROC curves to evaluate the predictive ability of the nomogram. I-K DCA curves determined that the nomogram can provide optimal clinical decision-making benefits. 
A

\begin{tabular}{|c|c|c|}
\multicolumn{1}{c|}{ TCGA cohort } \\
\hline predicted HCC & real HCC & real normal \\
\hline predicted normal & 46 & $\mathrm{l}$ \\
\hline totals & 5 & 49 \\
\hline correct & 46 & 50 \\
\hline sensitivity & 0.92 & 49 \\
\hline specificity & & 0.98 \\
\hline
\end{tabular}

E

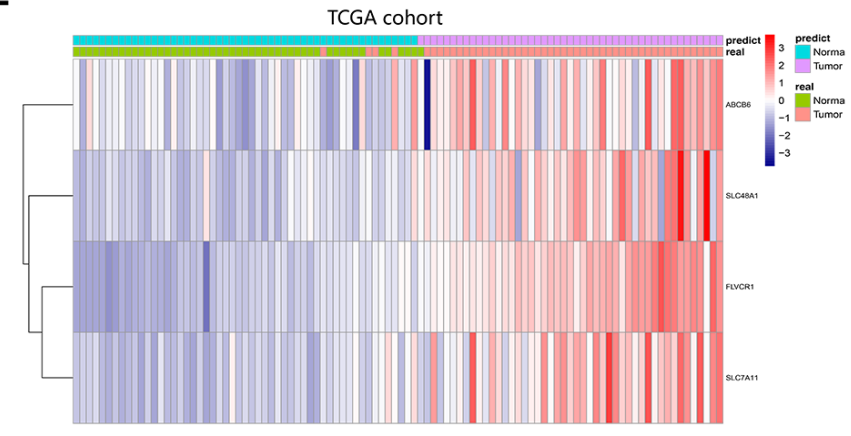

C

ICGC cohort

\begin{tabular}{|c|c|c|}
\hline & real HCC & real normal \\
\hline predicted HCC & 214 & 16 \\
\hline predicted normal & 29 & 186 \\
\hline totals & 243 & 202 \\
\hline correct & 214 & 186 \\
\hline sensitivity & 0.8807 & \\
\hline specificity & & 0.9208 \\
\hline
\end{tabular}

D
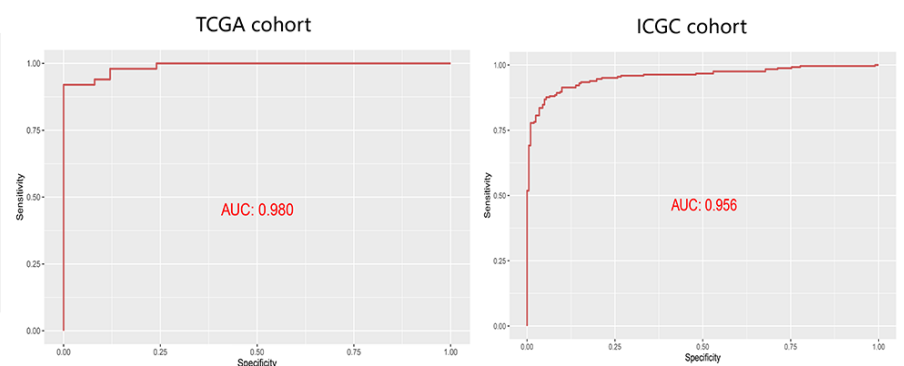

\section{$\mathbf{F}$}

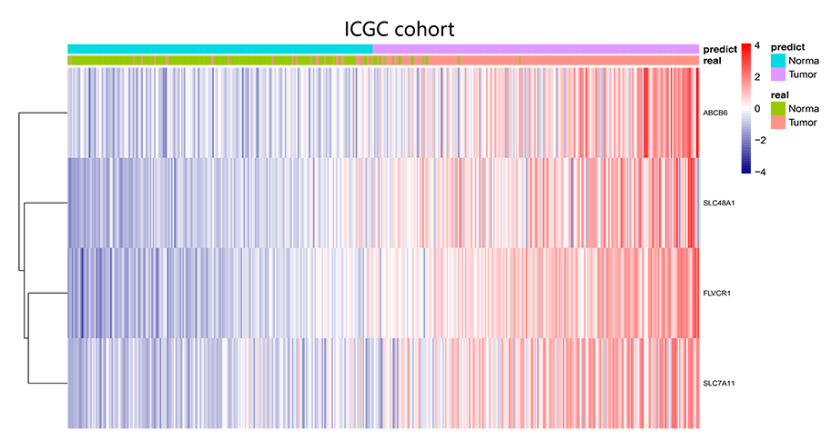

\section{Figure 4}

A diagnostic model for distinguishing HCC from normal samples in the HCC cohort from TCGA (A, C and $E)$ and ICGC (B, D and F). A and B Confusion matrix for the binary classification results of the diagnostic model. $C$ and D ROC curves for evaluating the predictive performance of the diagnostic model. $E$ and $F$ Unsupervised hierarchical clustering of the four ferroptosis- and iron metabolism-related genes for the diagnostic model.

A

\begin{tabular}{|} 
GSE6764 cohort \\
\begin{tabular}{|c|c|c|}
\hline & real HCC & real nodules \\
\hline predicted HCC & 34 & 1 \\
\hline predicted nodules & 1 & 16 \\
\hline totals & 35 & 17 \\
\hline correct & 34 & 16 \\
\hline sensitivity & 0.9714 & \\
\hline specificity & & 0.9412 \\
\hline
\end{tabular}
\end{tabular}

E

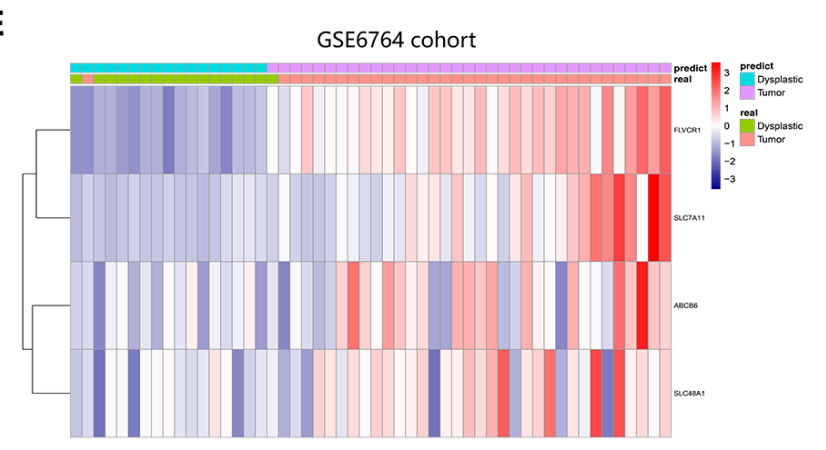

C

\begin{tabular}{|c|c|c|}
\hline \\
\hline & real HCC & real nodules \\
\hline predicted $\mathrm{HCC}$ & 39 & 11 \\
\hline predicted nodules & 10 & 13 \\
\hline totals & 49 & 24 \\
\hline correct & 39 & 13 \\
\hline sensitivity & 0.7959 & \\
\hline specificity & & 0.5417 \\
\hline
\end{tabular}

D

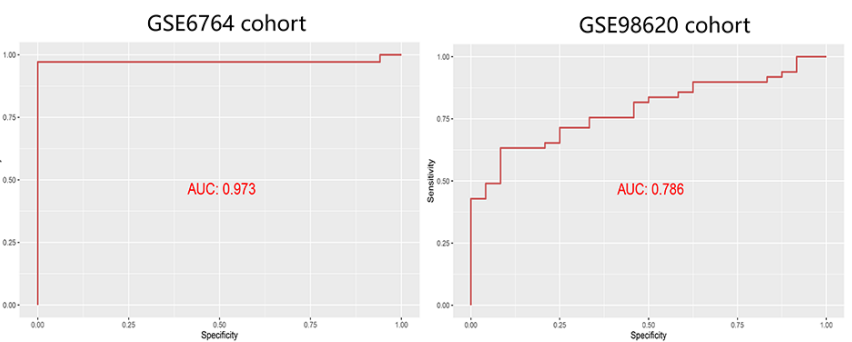

$\mathbf{F}$

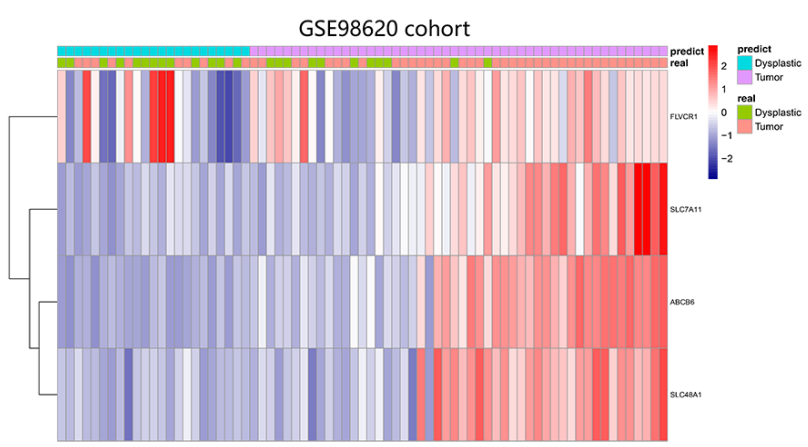

Figure 5 
A diagnostic model for distinguishing HCC from dysplastic nodules in the training dataset (GSE6764) (A, $\mathrm{C}$ and $\mathrm{E}$ ) and validation dataset (GSE98620) (B, D and F). A and B Confusion matrix for the binary classification results of the diagnostic model. $C$ and D ROC curves for evaluating the predictive performance of the diagnostic model. E and F Unsupervised hierarchical clustering of the four ferroptosisand iron metabolism-related genes for the diagnostic model.

A
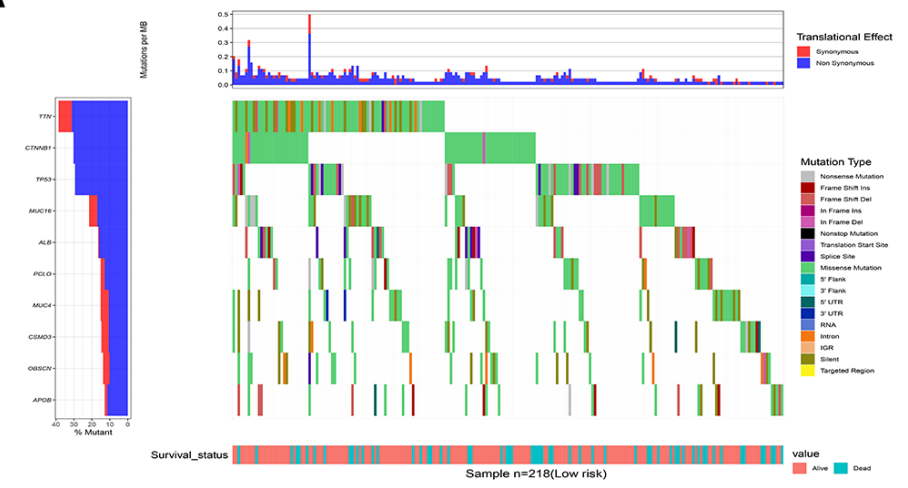

C

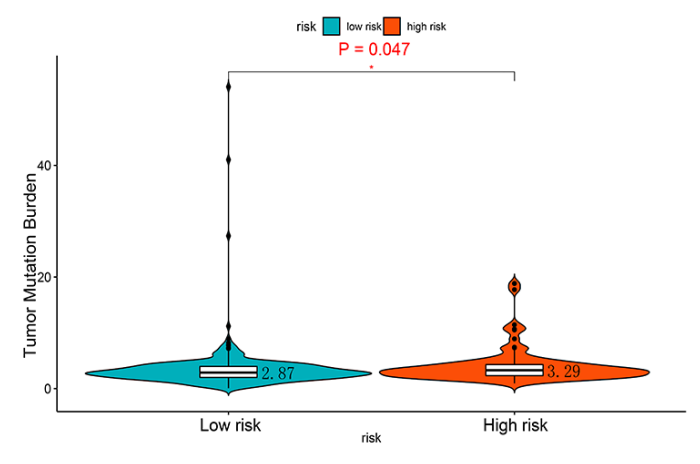

B

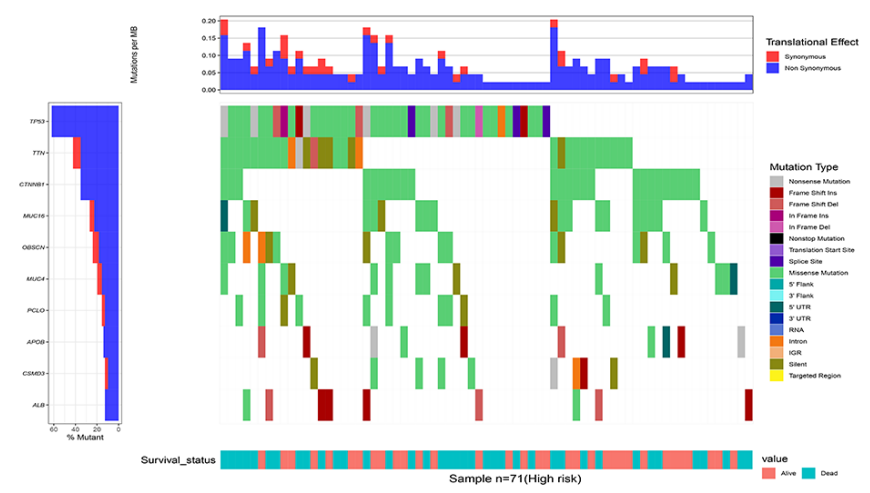

D

TCGA-LIHC

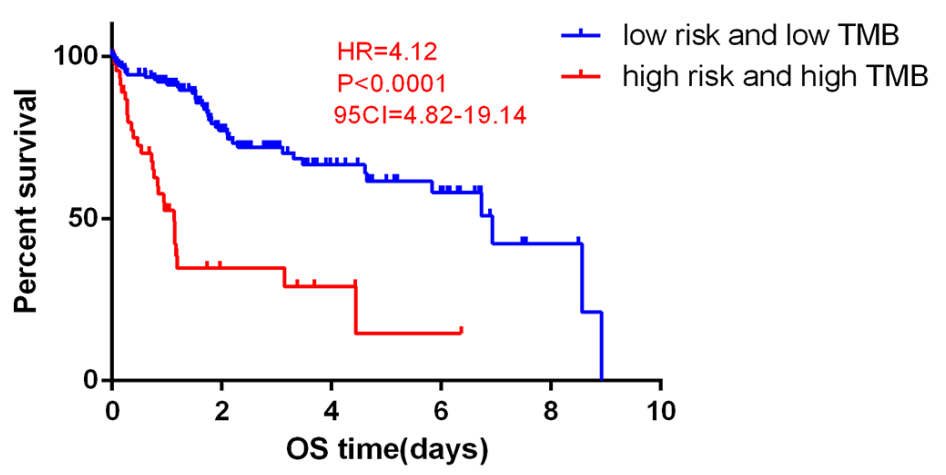

Figure 6

Correlations between risk scores and TMB and the predictive performance of TMB on OS. A-B The differences in TMB in somatic cells in patients with HCC between the high- and low-risk groups. C The high-risk group showed a higher TMB than the low-risk group. D OS rates in patients with low risk and low TMB were higher than those in patients with high risk and high TMB. 


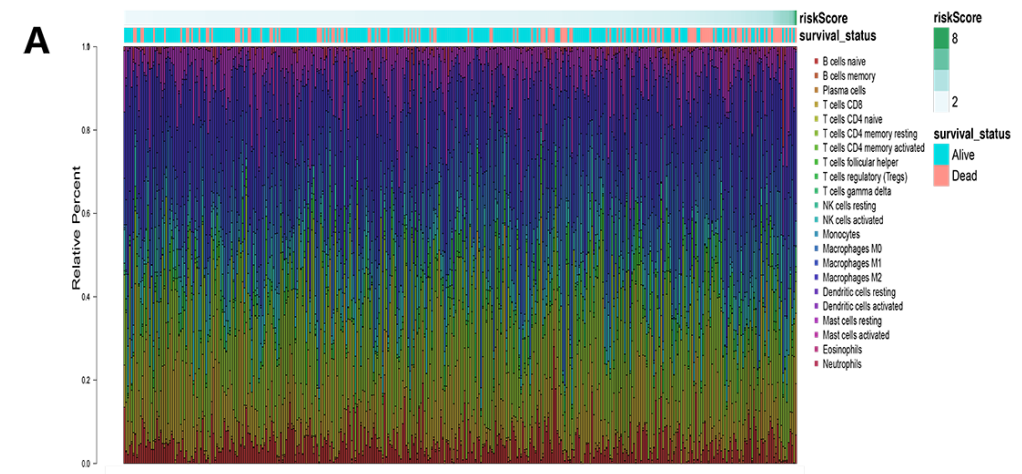

C
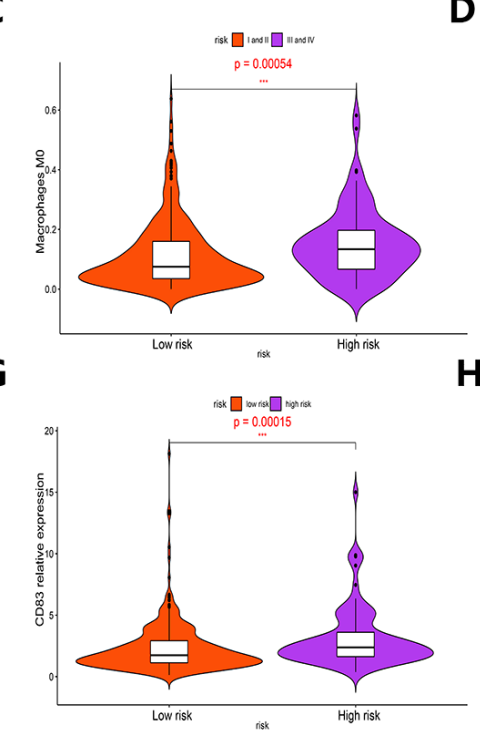

D
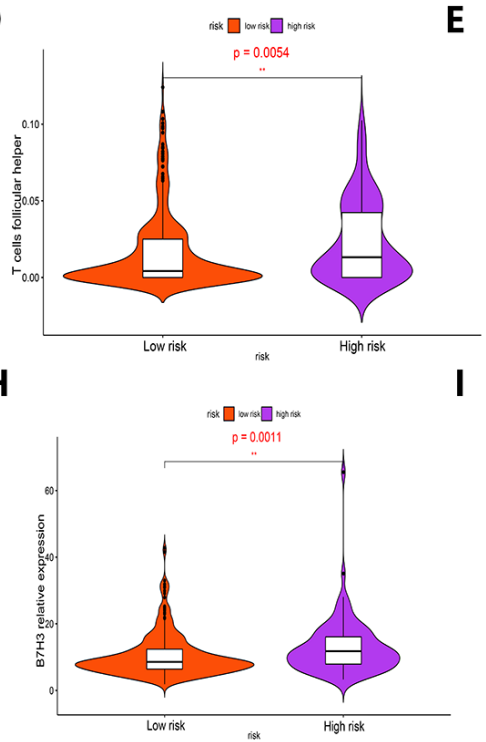

E
B
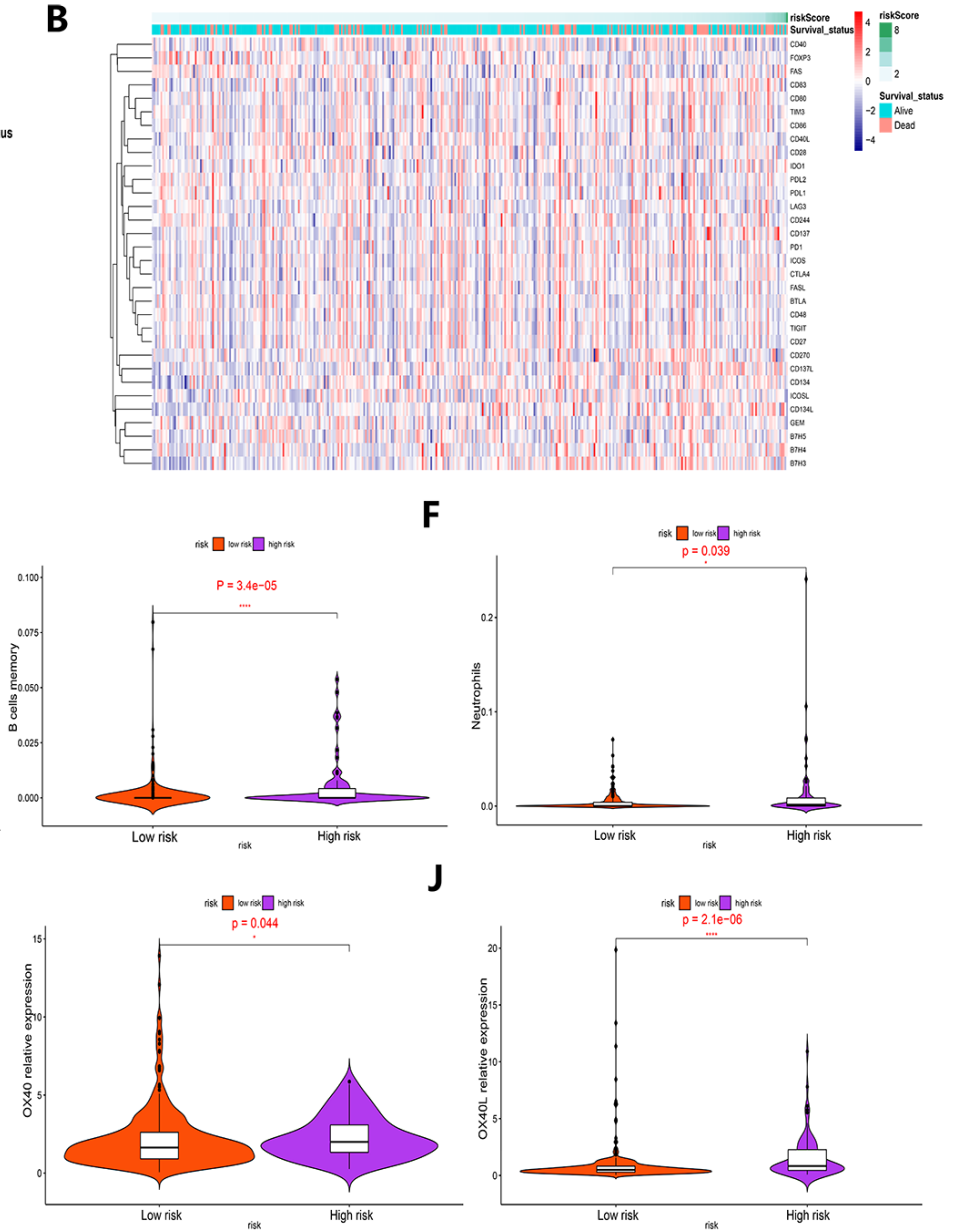

$\mathbf{F}$
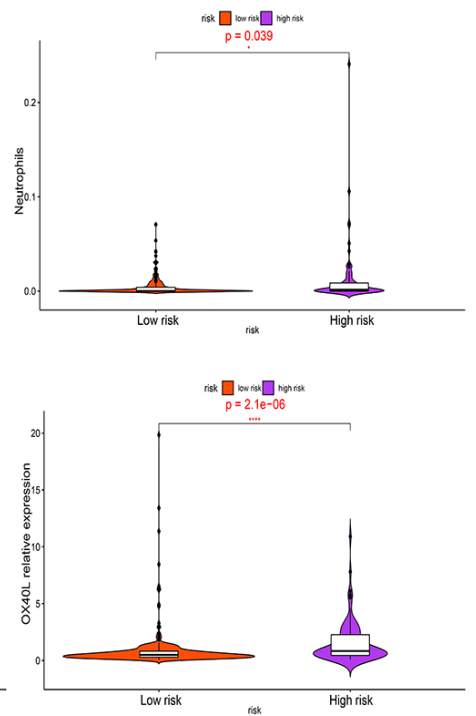

Figure 7

The landscape of immune infiltration and expression of immune checkpoints in patients with HCC with different risk scores. A The correlations between risk score and immune infiltration of 22 immune cell types in patients with HCC. B The relationship between the risk score and the expression of immune checkpoints. C-F Violin plots visualizing fractions of different immune cells in the high-risk and low-risk groups. G-J Violin plots visualizing the expression of immune checkpoints in the high-risk and low-risk groups. 
A

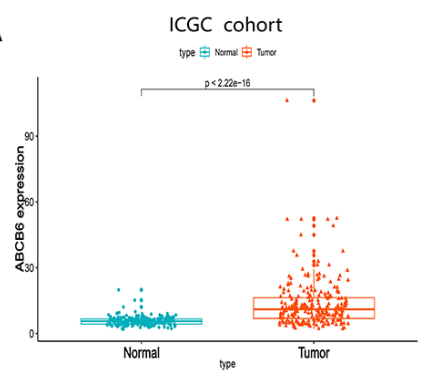

$\mathbf{E}$
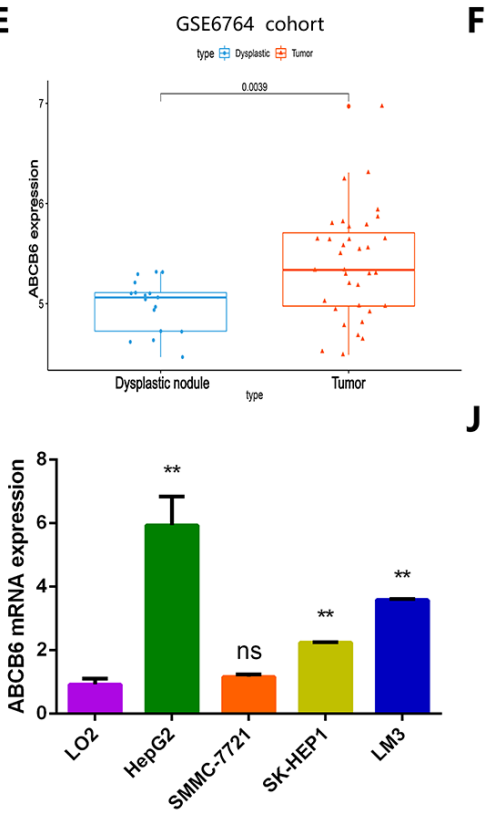

B

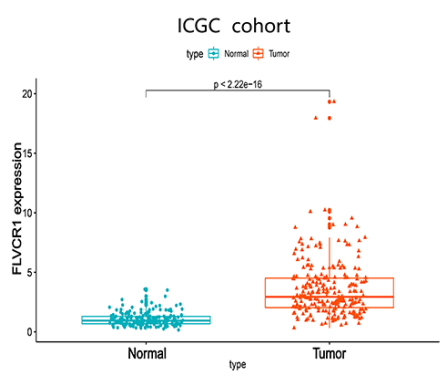

$\mathbf{F}$
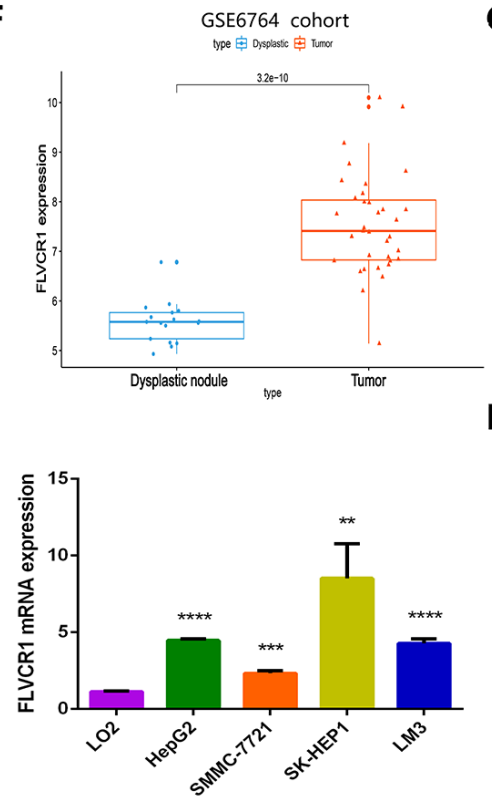

C

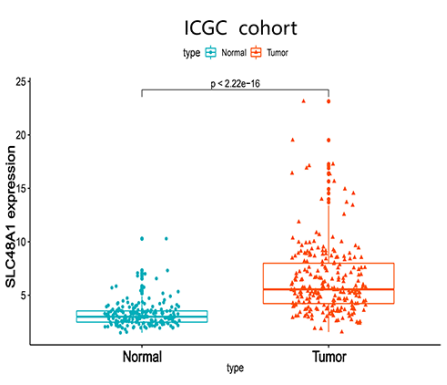

G
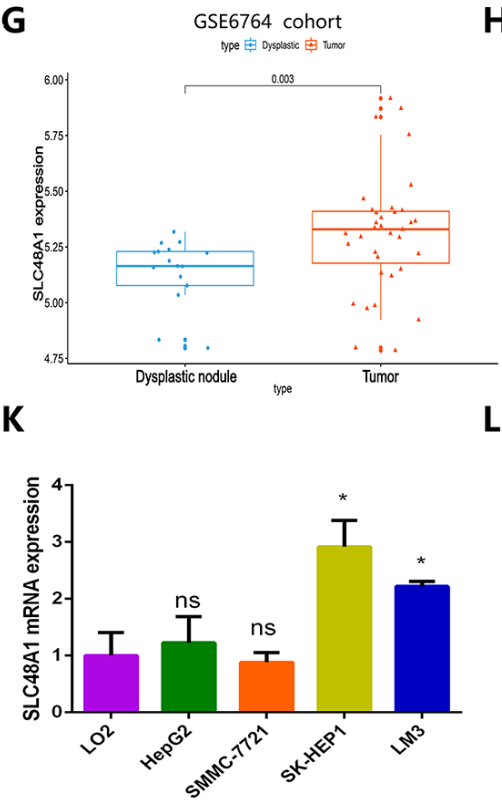

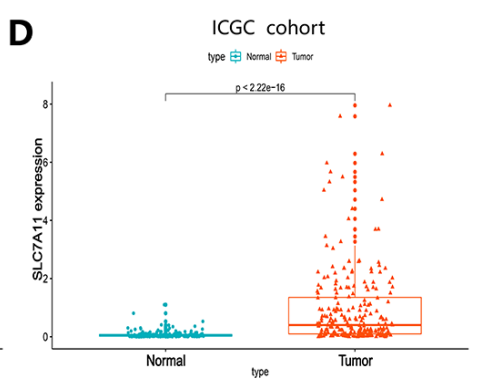

H GSE6764 cohort

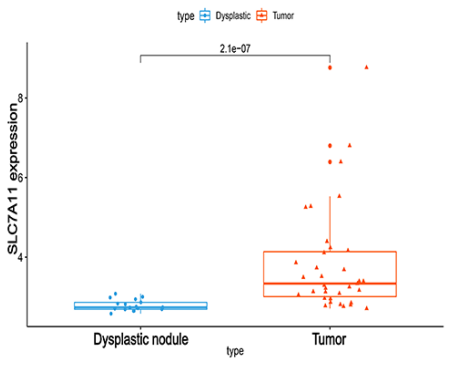

$\mathbf{L}$

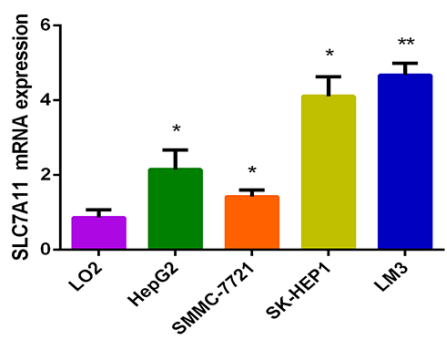

Figure 8

Validation of the expression patterns of the four ferroptosis- and iron metabolism-related genes. A-D Expression levels of the four genes in HCC and normal samples in the HCC cohort from ICGC. E-H Expression levels of the four genes in HCC and dysplastic nodule samples in the GSE6764 cohort. I-L The expression characteristics of the four genes in multiple types of HCC cell lines. 

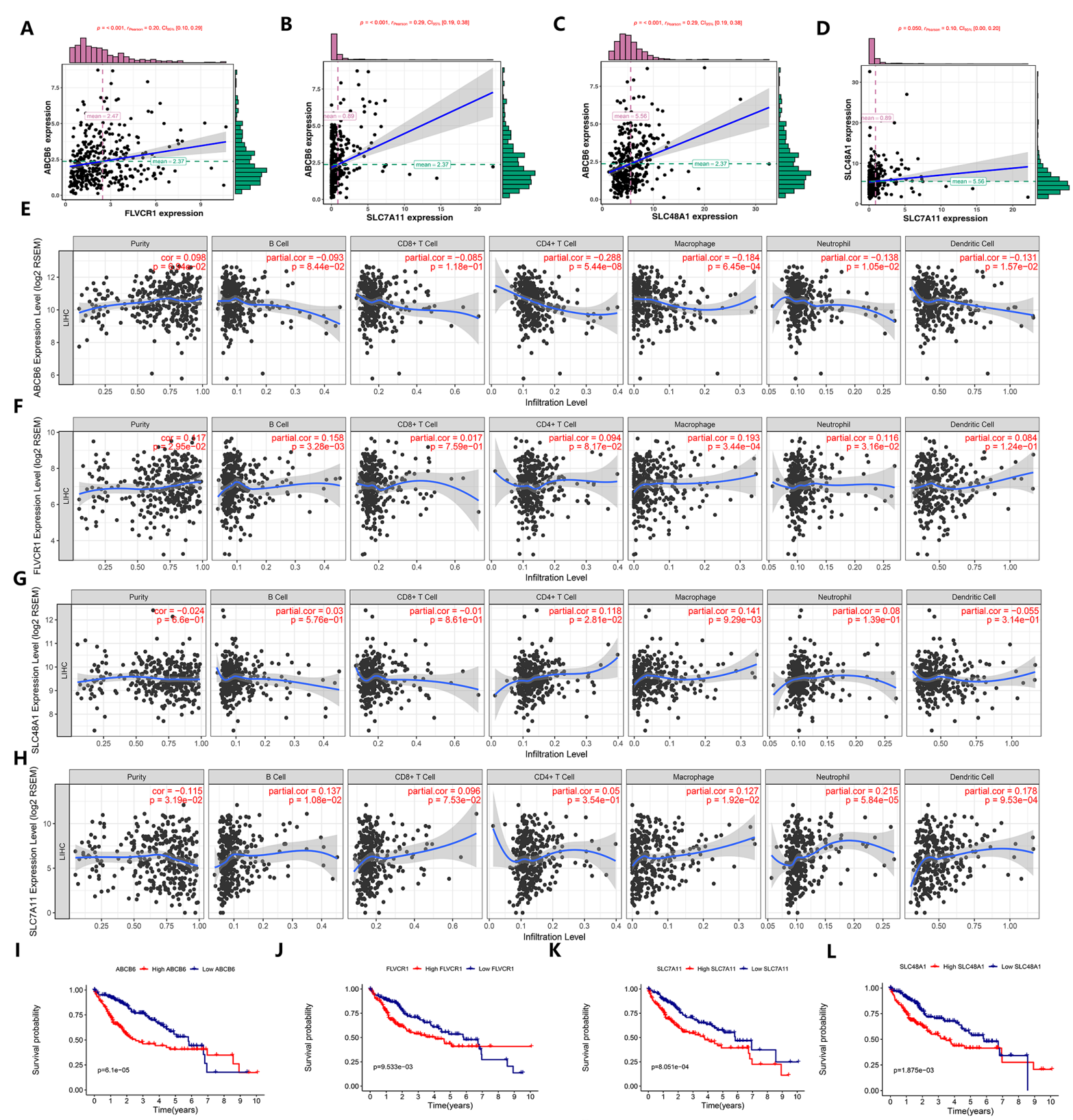

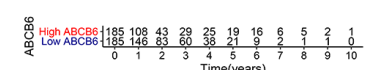

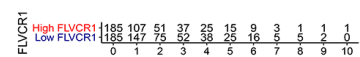

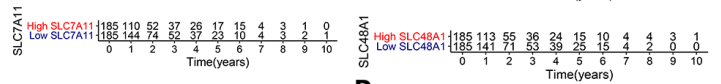
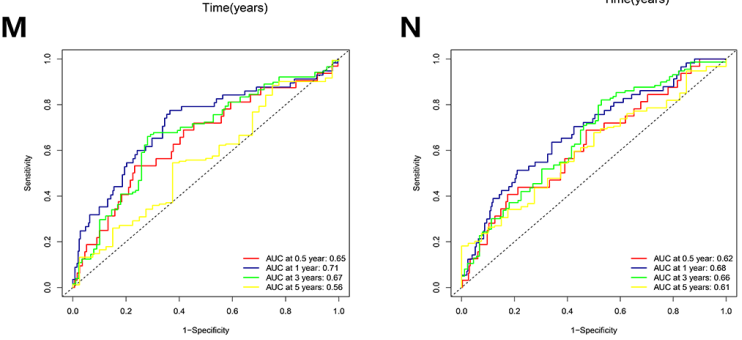

0

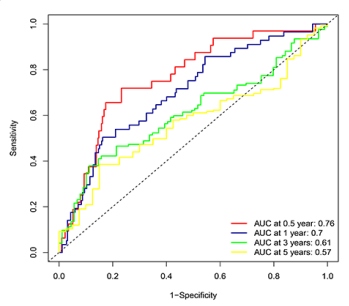

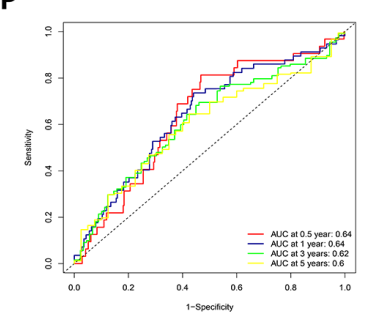

\section{Figure 9}

Regression analysis of expression levels among the four genes, correlations between genes and the density of the immune infiltrate, and predictive performance of genes on OS. A-D There was a synergistic effect among the expression levels of genes. E-H The impact of the expression of ABCB6 (E), FLVCR1 (F), SLC48A1 (G) and SLC7A11 ( $\mathrm{H})$ on infiltration by different immune cells. I-L K-M survival curves show the 
OS in the high-expression group and low-expression group. M-P Time-dependent ROC curve analysis for evaluating the predictive accuracy of the four genes for $0.5-, 1-, 3$ - and 5-year OS.

A

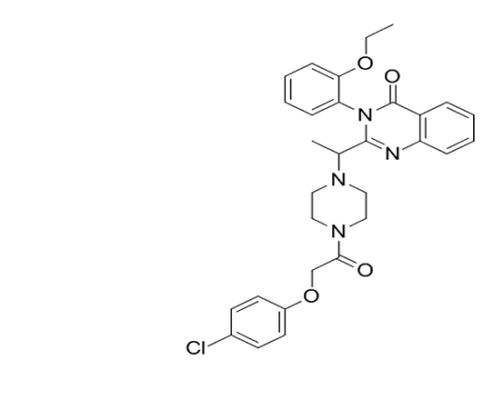

D

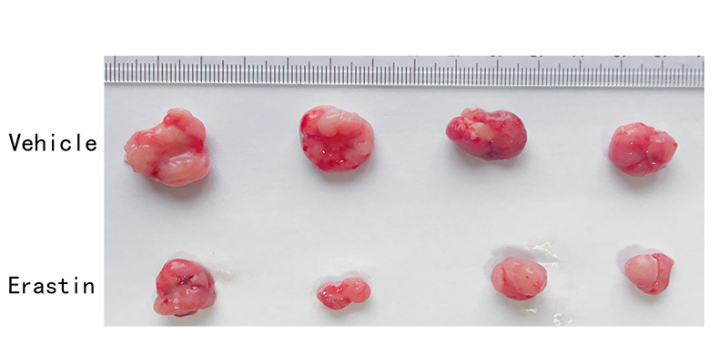

G

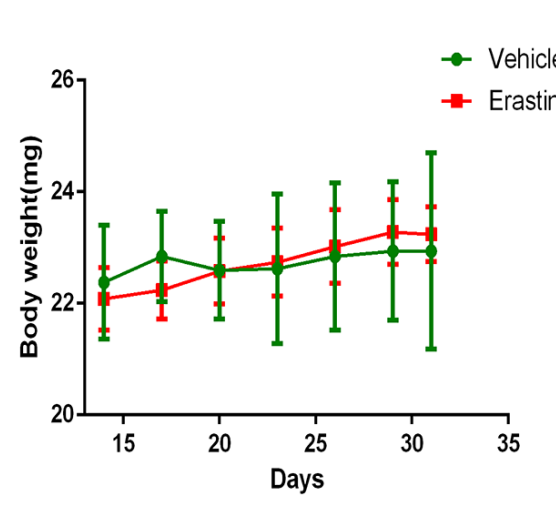

E

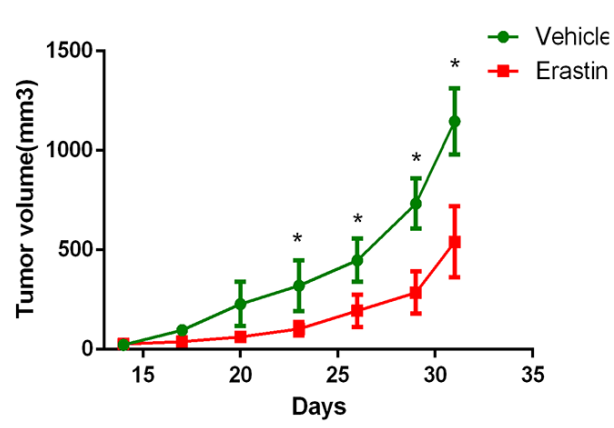

SK-HEP1

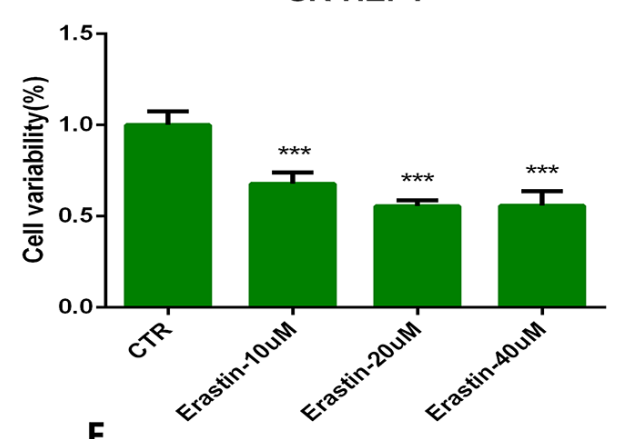

H

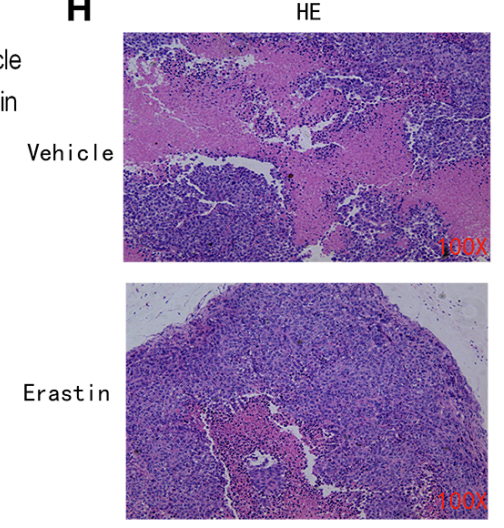

C

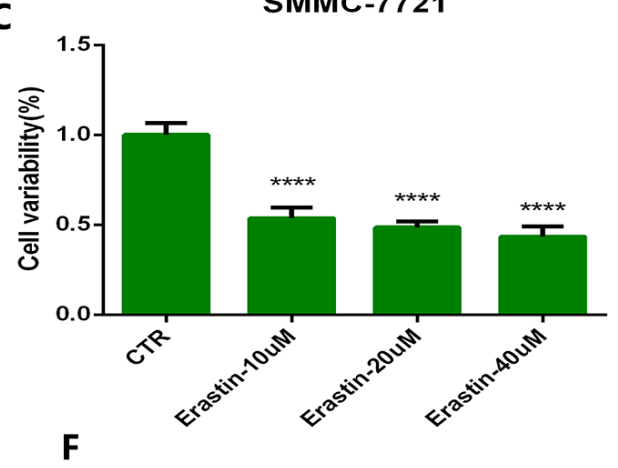

\section{Figure 10}
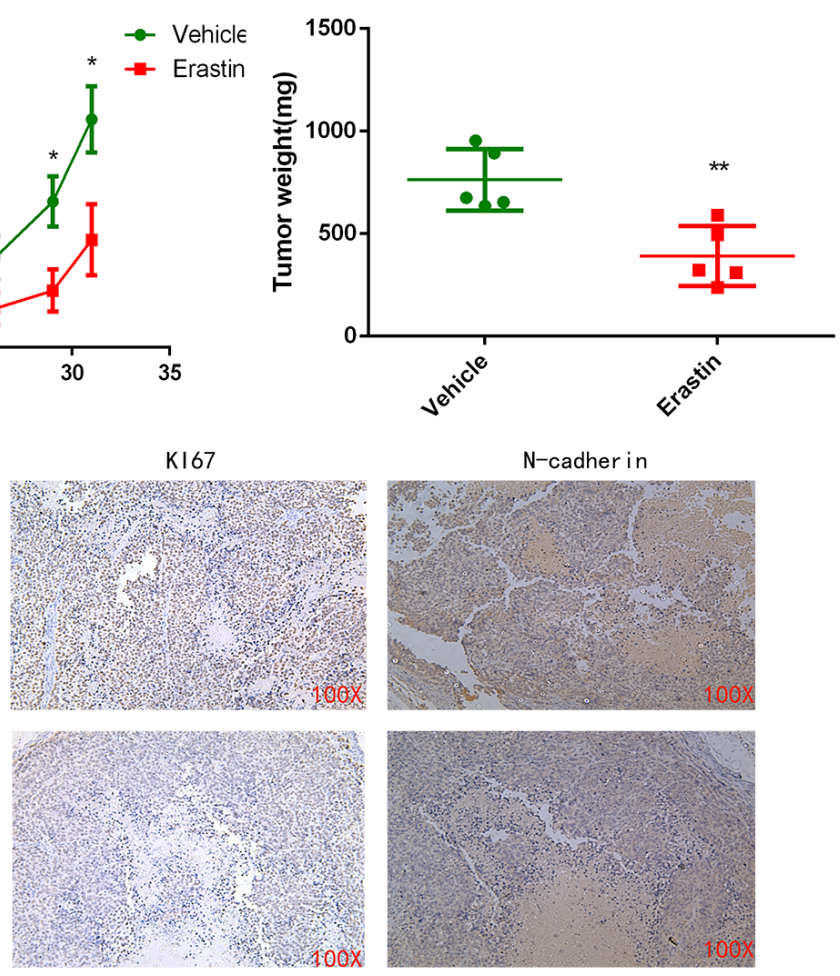

Inhibition by erastin of the proliferation and progression of HCC in vivo and in vitro. A The chemical formula of erastin. B-C The CCK-8 assay showed that erastin inhibited the proliferation of SK-HEP1 cells (A) and SMMC-7721 cells (B) in a dose-dependent manner. D-F The original tumors (D), tumor volume (E) and tumor weight $(F)$ under erastin treatment. $G$ Weight of mice under erstin treatment. H Pathological characteristics of tumor tissues and expression of Ki67 and N-cadherin in tumor tissues under erastin treatment. 
A

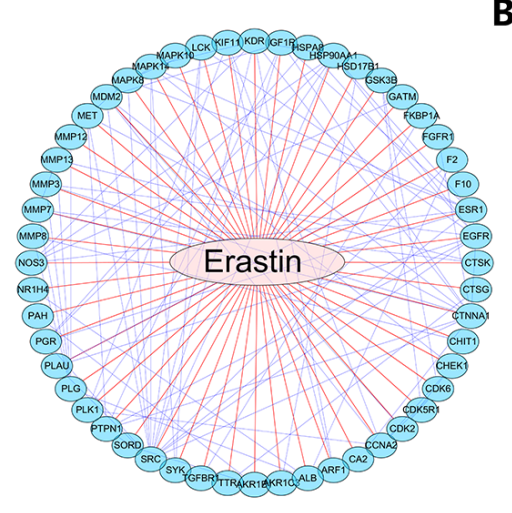

B

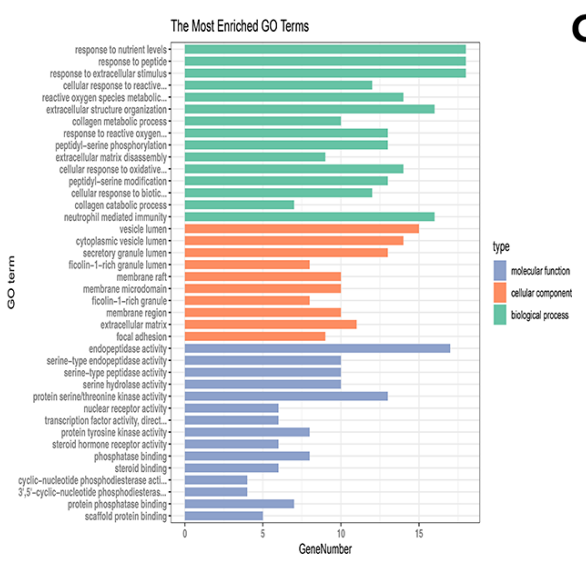

C

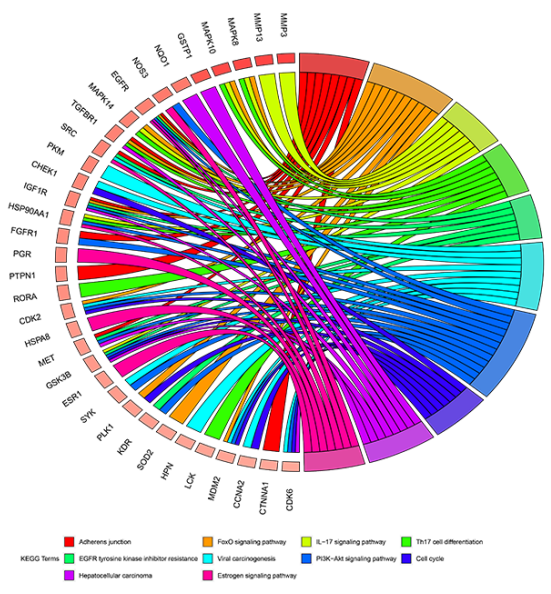

\section{Figure 11}

Possible molecular mechanism by which erastin inhibits the proliferation and progression of HCC. A The interaction of proteins regulated by erastin. B-C GO analysis (B) and KEGG pathway analysis (C) of the potential targets of erastin. 